TOPICAL REVIEW

\title{
Meromorphic solutions of difference equations, integrability and the discrete Painlevé equations
}

\author{
R G Halburd ${ }^{1}$ and R J Korhonen ${ }^{2}$ \\ ${ }^{1}$ Department of Mathematical Sciences, Loughborough University, Loughborough, \\ Leicestershire, LE11 3TU, UK \\ 2 Department of Mathematics, University of Joensuu, PO Box 111, FI-80101 Joensuu, Finland \\ E-mail: r.g.halburd@lboro.ac.uk and risto.korhonen@joensuu.fi
}

Received 29 September 2006, in final form 15 December 2006

Published 23 January 2007

Online at stacks.iop.org/JPhysA/40/R1

\begin{abstract}
The Painlevé property is closely connected to differential equations that are integrable via related iso-monodromy problems. Many apparently integrable discrete analogues of the Painlevé equations have appeared in the literature. The existence of sufficiently many finite-order meromorphic solutions appears to be a good analogue of the Painlevé property for discrete equations, in which the independent variable is taken to be complex. A general introduction to Nevanlinna theory is presented together with an overview of recent applications to meromorphic solutions of difference equations and the difference and $q$-difference operators. New results are presented concerning equations of the form $w(z+1) w(z-1)=R(z, w)$, where $R$ is rational in $w$ with meromorphic coefficients.
\end{abstract}

PACS numbers: $02.30 . \mathrm{Ik}, 02.30 . \mathrm{Ks}, 05.45 .-\mathrm{a}$

\section{Contents}

1. Introduction 2

2. Properties of discrete Painlevé equations 4

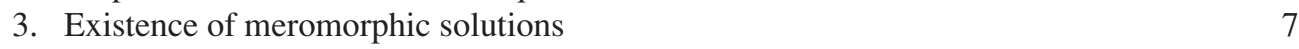

3.1. Linear difference equations $\quad 7$

3.2. Nonlinear first-order difference equations $\quad 7$

3.3. The QRT map 10

3.4. Nonlinear higher order difference equations 12

4. Nevanlinna theory $\quad 12$

4.1. Basic theory 13

4.2. Exceptional sets 14

4.3. Useful properties of the characteristic function $T(r, f) \quad 14$

1751-8113/07/060001+38\$30.00 @ 2007 IOP Publishing Ltd Printed in the UK R1 
5. Difference equations admitting finite-order meromorphic solutions 15

5.1. Difference equations of Painlevé type 16

5.2. Tools from Nevanlinna theory 19

5.3. The proof of theorem $5.5 \quad 21$

6. Linear difference equations 26

7. Value distribution of differences of meromorphic functions 28

7.1. Zeros of differences of meromorphic functions 28

7.2. Second main theorem of Nevanlinna theory 28

7.3. Second main theorem and Picard's theorem for the shift operator 29

7.4. Meromorphic functions sharing values $\quad 32$

8. $q$-difference equations $\quad 33$

9. Future work and conclusions $\quad 34$

Acknowledgments $\quad 35$

References

\section{Introduction}

Ordinary differential equations whose solutions have especially simple singularity structure are known to have remarkable integrability properties. The Painlevé equations are particularly important in this context. These six equations (denoted $P_{\mathrm{I}}-P_{\mathrm{VI}}$ ) possess the Painlevé property, i.e., all movable singularities of all solutions are poles, and have been known for over a century. Each of the Painlevé equations can be written as the compatibility condition for a related linear problem, a so-called iso-monodromy problem, which underlies the integrability of the equation.

The discrete equation

$$
x_{n+1}+x_{n}+x_{n-1}=\frac{a n+b}{x_{n}}+c
$$

where $a, b$ and $c$ are constants, appeared in studies of a partition function in quantum gravity $[12,17,32,41,101]$. It was realized that equation (1) is very special, in particular, it is the compatibility condition for a linear problem, which was studied in [70], and it has a simple continuum limit to the first Painlevé equation, $P_{\mathrm{I}}[17,25,41]$. It would appear natural to call equation (1) a discrete Painlevé equation. Equation (1) had previously appeared in works on the coefficients in the three-point recurrence relations of certain classes of orthogonal polynomials [33, 71, 122]. It was Magnus [84] who explicitly noted that a discrete Painlevé equation arises in this context.

Soon other discrete Painlevé equations were discovered as reductions of integrable lattice equations [94] and by looking for discrete isomonodromy problems [31, 74]. But what exactly is a discrete Painlevé equation? More fundamentally, is there a discrete analogue of the Painlevé property?

The first potential analogue of the Painlevé property for discrete equations to appear explicitly as such in the literature is the singularity confinement property of Grammaticos, Ramani and Papageorgiou [38]. The singularity confinement test involves considering the behaviour of a sequence of iterates $x_{n}$ of an equation such as (1) that goes through a singularity of the equation. In the case of equation (1), this means considering a sequence of iterates such that $x_{n-1}$ is an arbitrary finite value and $x_{n}=0$, in which case $x_{n+1}=\infty$. In order to continue the solution further it is convenient to consider the perturbed problem $x_{n-1}=k$ and $x_{n}=\epsilon$. After calculating any number of iterates, we then take the limit $\epsilon \rightarrow 0$. For generic equations, 
the list of future iterates appears to include infinitely many singular points. However, for equation (1), the singularity appears to be confined (i.e., future iterates are finite.)

Singularity confinement is a powerful tool which has been used to discover many discrete Painlevé equations. However, Hietarinta and Viallet found an example [61] of a simple equation that appears to possess the singularity confinement property but is chaotic. They suggested that, in order to exclude such non-integrable examples, singularity confinement should be augmented by the condition that the equation has zero algebraic entropy. Zero algebraic entropy corresponds to the case in which the degree of the $n$th iterate of a discrete equation, considered as a rational function of the initial conditions, is bounded by a power of $n[7,29]$. Veselov [126] showed that exponential growth of the degree of iterates (which is generic) corresponds to the non-existence of certain types of first integrals. Orbit dynamics over finite fields have been considered by Roberts and Vivaldi [113].

In [1], Ablowitz, Halburd and Herbst looked for a purely complex analytic analogue of the Painlevé property for discrete equations. In order to do this, discrete equations such as (1) were reinterpreted as difference equations such as

$$
y(z+1)+y(z)+y(z-1)=\frac{a z+b}{y(z)}+c,
$$

for all $z \in \mathbb{C}$. A solution of equation (1) is a sequence $\left(x_{n}\right)$ which, given initial conditions $x_{0}$ and $x_{1}$, can be constructed iteratively. In contrast, a solution of equation (2) is a function $y: \mathbb{C} \rightarrow \mathbb{C P}^{1}$. There is no unique way of extending a solution of equation (1), which 'lives' on the integers, to a solution of equation (2), which 'lives' on $\mathbb{C}$. In our analysis of the analytic properties of solutions of difference equations, we do not consider these solutions as arising from solutions of discrete equations. For ideas related to extensions to analysable functions, see Costin and Kruskal [24].

An initial value problem for equation (2) involves specifying $y$ on, for example, the infinite strip $S=\left\{z: z_{0}-1 \leqslant z<z_{0}+1\right\}$. In general, a solution $y$ of equation (2) that is meromorphic on $S$ is not meromorphic on $\mathbb{C}$-it will have discontinuities on the lines $\operatorname{Re}(z)=z_{0}+2 n+1, n \in \mathbb{Z}$. The existence of meromorphic solutions of difference equations is poorly understood, yet classes of equations (such as first-order rational difference equations), including non-integrable equations, are known to admit large families of meromorphic solutions. In [1] it was suggested that a useful analogue of the Painlevé property for difference equations is the existence of sufficiently many meromorphic solutions of finite order in the sense of Nevanlinna theory.

It was observed independently by Osgood [97, 99] and Vojta [127] that there is a strong parallel between Nevanlinna theory and Diophantine approximation. The Nevanlinna approach to difference equations of Painlevé type suggests a parallel number theoretical approach to discrete equations called Diophantine integrability [46]. As well as being very easy to test numerically, this property suggests that there may be Diophantine interpretations of the discrete Painlevé equations.

This review will concentrate on using Nevanlinna theory to detect integrable difference equations. It should be stressed that this is just one of several approaches to the integrability of difference/discrete equations. There are a number of different notions of 'integrable' or 'Painlevé' in the literature, leading to slightly different classifications of equations. Yet these different approaches often agree on certain canonical examples. One of the main purposes of studying the existence of finite-order meromorphic solutions is that, as is the case for the genuine (differential) Painlevé property, this is a well-defined complex analytic property which allows us to classify and study equations without getting bogged down with the question of what exactly one means by integrability. Results such as theorem 5.4 suggest that this property singles out equations that are known to have more classical indicators of integrability such 
as the existence of related linear problems, Bäcklund transformations, special solutions and relations to lattice soliton equations.

For the most part we will be concerned with meromorphic solutions of nonlinear equations. Linear equations will only be discussed in the context of recent results from Nevanlinna theory. For example, we will not describe the rich isomonodromy theory of Birkhoff $[13,14]$.

The outline of this review is as follows. In section 2 we will describe, by way of examples, some of the many remarkable properties of discrete Painlevé equations mentioned above, such as related linear problems and the derivation of discrete equations from the Bäcklund transformations of (differential) Painlevé equations. In section 3 we will survey a number of results on the existence of non-constant meromorphic solutions of difference equations. We outline a simple direct proof for the existence of meromorphic solutions in the first-order case. We also describe the Quispel-Roberts-Thompson (QRT) map, which is a very general class of second-order maps closely related to the discrete Painlevé equations. Much work remains to be done on the existence of meromorphic solutions in the case of general rational difference equations of order 2 or more.

Section 4 contains an introduction to basic Nevanlinna theory, in particular, we describe some simple properties of the Nevanlinna characteristic and the lemma on the logarithmic derivative. Nevanlinna theory is applied to difference equations in section 5. We describe how to find strong necessary conditions for an equation to admit a meromorphic solution of finite order. We state theorem 5.4, which describes equations of the form $w(z+1)+w(z-1)=$ $R(z, w(z))$, where $R$ is rational in $w$ with meromorphic coefficients, which can admit solutions of finite order. We illustrate the ideas involved by considering a new example of an equation of the form $w(z+1) w(z-1)=R(z, w(z))$, leading to a well-known integrable difference equation often called $d P_{\mathrm{III}}$. Some of the main tools used in this section are difference analogues of the lemma of the logarithmic derivative, Clunie's lemma and a value distribution result of Mohon'ko and Mohon'ko.

In section 6 we describe a number of recent results on meromorphic solutions of linear difference equations. In particular we describe a version of Wiman-Valiron theory for slow growing functions due to Ishizaki and Yanagihara, order estimates for the growth of finiteorder solutions by Chiang and Feng, and a theorem concerning minimal solutions of first-order equations by Chiang and Ruijsenaars. Section 7 contains a number of results concerning the value distribution of differences of meromorphic functions. Some of these results grew out of tools developed in the classification of equations admitting finite-order meromorphic solutions. In particular we describe difference analogues of the Nevanlinna's second main theorem, Picard's theorem, defect relations and theorems concerning meromorphic functions sharing values. Other results by Bergweiler and Langley may be useful in the study of slow growth meromorphic solutions of difference equations. In section 8 we describe a $q$-difference analogue of the lemma on the logarithmic derivative from [4] which leads to $q$-difference analogues of some of the theorems mentioned above. Finally section 9 lists a number of important outstanding problems in the field.

\section{Properties of discrete Painlevé equations}

There are many discrete equations that are considered to be integrable discrete analogues of the Painlevé equations. Each of these equations is known to possess at least some of the following remarkable properties: the existence of a related linear (iso-monodromy) problem, the singularity confinement property, the existence of Bäcklund transformations, relations to Bäcklund transformations of differential Painlevé equations, zero algebraic entropy, Diophantine integrability. Although there is a vast literature devoted to the discrete 
Painlevé equations, most of the results involve looking at only one or two properties of a small number of equations. At present there is no global theory of the discrete Painlevé equations which explains how all of these properties are related, although the algebro-geometric approach of Sakai [118], which builds on earlier work of Okamoto, might provide the starting point for such an undertaking. At the moment we cannot answer some very simple questions such as do all of the discrete Painlevé equations have Lax pairs (related linear problems). If so, how do we use these Lax pairs to describe solutions? Are all the discrete Painlevé equations related to Bäcklund transformations of other kinds of integrable equations?

This section has a very modest objective. We begin by deriving a well-known difference equation from the addition law of the Weierstrass $\wp$ function. We will then concentrate on a non-autonomous deformation of this equation, which possesses a continuum limit to the first Painlevé equation. Following Fokas, Grammaticos, and Ramani [31], we will show that this equation is the compatibility condition for a related linear problem and that it describes the Bäcklund transformations of a special case of the third Painlevé (differential) equation. The purpose of this section is to emphasize the importance from the point of view of integrable systems of the kinds of equations to which we are led in later sections by considering special complex-analytic properties.

Let $\omega_{1}$ and $\omega_{2}$ be two complex numbers such that $\operatorname{Im}\left(\omega_{2} / \omega_{1}\right) \neq 0$. The Weierstrass $\wp$ function is defined by

$$
\wp(z):=\frac{1}{z^{2}}+\sum_{m, n}^{\prime}\left\{\frac{1}{\left(z-\Omega_{m n}\right)^{2}}-\frac{1}{\Omega_{m n}^{2}}\right\},
$$

where $\Omega_{m n}=2\left(m \omega_{1}+n \omega_{2}\right)$ and $\sum_{m, n}^{\prime}$ denotes the sum over all integer $m$ and $n$ excluding $(m, n)=(0,0)$. The Weierstrass $\wp$ function is an even meromorphic function with periods $2 \omega_{1}$ and $2 \omega_{2}$ and satisfies the ODE

$$
\wp^{\prime 2}=4 \wp^{3}-g_{2} \odot-g_{3},
$$

where

$$
g_{2}:=60 \sum_{m, n}^{\prime} \Omega_{m n}^{-4} \quad \text { and } \quad g_{3}:=140 \sum_{m, n}^{\prime} \Omega_{m n}^{-6} .
$$

The Weierstrass $\wp$ function has order 2 in the sense of Nevanlinna (see section 4.) An important property of $\wp$ is the addition law,

$$
\wp\left(z_{1}+z_{2}\right)=\frac{1}{4}\left\{\frac{\wp^{\prime}\left(z_{1}\right)-\wp^{\prime}\left(z_{2}\right)}{\wp\left(z_{1}\right)-\wp\left(z_{2}\right)}\right\}^{2}-\wp\left(z_{1}\right)-\wp\left(z_{2}\right) .
$$

Using this addition law, together with equation (4) and the fact that $\wp$ is even, it can be seen that, for fixed $h$ not a pole of $\wp, y(z):=\wp\left(h\left(z-z_{0}\right)\right)-\wp(h)$ satisfies

$$
y(z+1)+y(z-1)=\frac{\alpha y(z)+\beta}{y^{2}(z)},
$$

where $\alpha=\left(12 \wp^{2}(h)-g_{2}\right) / 2$ and $\beta=\wp^{\prime 2}(h)$. This is a two-parameter $\left(z_{0}\right.$ and $\left.g_{3}\right)$ family of solutions. Therefore, equation (6) admits a two-parameter family of finite-order meromorphic solutions. More generally, $z_{0}$ and $g_{3}$ can be taken to be arbitrary period 1 functions, however, care must be taken if $y$ is required to be meromorphic.

Each of the six Painlevé equations is known to be the compatibility condition of a linear (iso-monodromy) problem of the form $\Psi_{z}=A(z, \zeta) \Psi, \Psi_{\zeta}=B(z, \zeta) \Psi$, for suitable choices of matrix-valued functions $A$ and $B$, where $z$ is the independent variable of the Painlevé equation and $\zeta$ is a (spectral or iso-monodromy) parameter, external to the Painlevé equation itself. A natural difference analogue of this is the system

$$
\Psi(z+1, \zeta)=U(z, \zeta) \Psi(z, \zeta), \quad \partial_{\zeta} \Psi(z, \zeta)=V(z, \zeta) \Psi(z, \zeta)
$$


In terms of the $z$-shift operator $E_{z}$, defined by $E_{z} \Psi(z, \zeta):=\Psi(z+1, \zeta)$, the compatibility of the system (7) is $E_{z} \partial_{\zeta} \Psi(z, \zeta)=\partial_{\zeta} E_{z} \Psi(z, \zeta)$. This is equivalent to the condition

$$
\partial_{\zeta} U(z, \zeta)=V(z+1, \zeta) U(z, \zeta)-U(z, \zeta) V(z, \zeta)
$$

Fokas, Grammaticos and Ramani [31] consider the particular choice

$$
U(z, \zeta):=\left(\begin{array}{cc}
2 \zeta / \Omega(z+1) & -\Omega(z) / \Omega(z+1) \\
1 & 0
\end{array}\right)
$$

for some function $\Omega$. If $\Psi(z, \zeta)=\left(\psi_{1}(z, \zeta), \psi_{2}(z, \zeta)\right)^{T}$, then it follows from the first equation in (7) that $\psi_{1}$ satisfies

$$
\Omega(z+1) \psi_{1}(z+1, \zeta)+\Omega(z) \psi_{1}(z-1, \zeta)=2 \zeta \psi_{1}(z, \zeta)
$$

which is a difference version of the Schrödinger equation. Assume that $\operatorname{Tr}(V)=0$. Then the compatibility condition (8) shows that $V$ has the form

$$
V(z, \zeta)=\left(\begin{array}{cc}
a(z, \zeta) & -\frac{\Omega(z)}{2 \zeta}[a(z, \zeta)+a(z+1, \zeta)] \\
\frac{\Omega(z)}{2 \zeta}[a(z, \zeta)+a(z-1, \zeta)] & -a(z, \zeta)
\end{array}\right)
$$

where, on writing $a(z) \equiv a(z, \zeta)$, we have

$\zeta[a(z+1)-a(z)]-\Omega(z+1)^{2} \frac{a(z+1)+a(z+2)}{4 \zeta}+\Omega(z)^{2} \frac{a(z-1)+a(z)}{4 \zeta}=1$.

Seeking a solution of equation (10) of the form $a(z, \zeta)=\phi(z) / \zeta+2 \beta(z) \zeta$, gives

$$
\phi(z)=\beta \Omega^{2}(z)+z+\alpha, \quad \beta(z)=\beta,
$$

where $\alpha$ and $\beta$ are constants, and

$$
[\phi(z-1)+\phi(z)] \Omega(z)^{2}-[\phi(z+1)+\phi(z+2)] \Omega(z+1)^{2}=0 .
$$

Multiplying equation (11) by the integrating factor $\phi(z)+\phi(z+1)$ and 'integrating' the resulting exact difference gives

$$
(\phi(z)+\phi(z+1))(\phi(z)+\phi(z-1))=\frac{\gamma}{\phi(z)-z-\alpha},
$$

where $\gamma$ is an arbitrary period 1 function, which we take to be constant. Putting $y(z)=1 /(\phi(z)+\phi(z-1))$ in equation (12) gives

$$
y(z+1)+y(z-1)=\frac{1-(2 z+2 \alpha-1) y(z)}{\gamma y^{2}(z)},
$$

which is a non-autonomous generalization of equation (6) with a continuum limit to the first Painlevé equation.

The following example in which a discrete Painlevé equation is constructed from Bäcklund transformations of a differential Painlevé equation is also from Fokas, Grammaticos and Ramani [31]. The third Painlevé equation is

$$
w^{\prime \prime}=\frac{w^{\prime 2}}{w}-\frac{w^{\prime}}{x}+\frac{1}{x}\left(\alpha w^{2}+\beta\right)+\gamma w^{3}+\frac{\delta}{w},
$$

where $\alpha, \beta, \gamma$ and $\delta$ are constants. If $\gamma=0$ but $\alpha$ and $\delta$ are non-zero, then we rescale $x$ and $w$ such that $\alpha=\delta=-1$ in the transformed equation, which takes the form

$$
w^{\prime \prime}=\frac{w^{\prime 2}}{w}-\frac{w^{\prime}}{x}-\frac{1}{x}\left(w^{2}-\beta\right)-\frac{1}{w} .
$$

If $w \equiv w(x, \beta)$ is a solution of equation (14) then 


$$
\begin{aligned}
& w(x ; \beta+2)=\frac{x\left(1+w^{\prime}\right)}{w^{2}}-\frac{\beta+1}{w}, \quad \text { and } \\
& w(x ; \beta-2)=\frac{x\left(1-w^{\prime}\right)}{w^{2}}-\frac{\beta-1}{w},
\end{aligned}
$$

are also solutions with $\beta$ replaced by $\beta+2$ and $\beta-2$, respectively. Adding equations (15) and (16) gives

$$
w(x ; \beta+2)+w(x ; \beta-2)=\frac{2 x}{w^{2}(x ; \beta)}-\frac{2 \beta}{w(x ; \beta)} .
$$

For fixed $x$, let $y(z)=A w(x ; 2 z+B)$, where $A \neq 0$ and $B$ are constants. Then $y$ satisfies

$$
y(z+1)+y(z-1)=\frac{(a z+b) y(z)+c}{y^{2}(z)},
$$

where $a=-4 A^{2} \neq 0, b=-2 A^{2} B$ and $c=2 x A^{3}$, which is a well-known discrete Painlevé equation which is equivalent to equation (13).

\section{Existence of meromorphic solutions}

In this section we present a number of theorems on the existence of meromorphic solutions of certain classes of difference equations, some of which we will study in more detail in sections 5 and 7. For a more comprehensive account on the existence and analytic properties of solutions of difference equations, including asymptotic behaviour and formal solutions, we refer to the monographs $[5,65,87,88,95,128]$.

\subsection{Linear difference equations}

Nörlund [95] has shown that the linear difference equation

$$
a_{n}(z) w(z+n)+\cdots+a_{1}(z) w(z+1)+a_{0}(z) w(z)=0
$$

with entire coefficients $a_{0}(z), \ldots, a_{n}(z)$ (satisfying certain growth conditions as $\operatorname{Re}(z) \rightarrow \infty$ ) has a system of $n$ meromorphic solutions on $\mathbb{C}$, linearly independent over the periodic functions. Praagman [103] applied a method due to Röhrl [114] to give an alternative proof for the existence of meromorphic solutions by considering the solutions of (18) as sections of a sheaf of modules over the sheaf of meromorphic periodic functions.

Theorem 3.1 $[95,103]$. The equation $w(z+1)=A(z) w(z)$, with A a meromorphic invertible $n \times n$ matrix function on $\mathbb{C}$, admits a fundamental solution $W$, a meromorphic $n \times n$ invertible matrix function on $\mathbb{C}$.

\subsection{Nonlinear first-order difference equations}

Harris and Sibuya have studied the system of first-order difference equations

$$
\vec{w}(z+1)=\vec{F}(z, \vec{w}(z)),
$$

where $\vec{w}$ and $\vec{F}$ are $n$-dimensional vectors [51-53]. They showed that if $\vec{F}(\infty, \overrightarrow{0})=\overrightarrow{0}$ and the components of $\vec{F}$ are holomorphic in a certain region, then equation (19) has a holomorphic solution in a suitable angular domain. Although their method works for large classes of difference equations, proving the global existence of non-trivial meromorphic solutions of difference equations in the complex plane requires a different approach. Fatou [30], and later 
Shimomura proved the existence of entire solutions of a large class of first-order difference equations.

Theorem 3.2 (Shimomura [121]). For any non-constant polynomial $P(w)$, the difference equation

$$
w(z+1)=P(w)
$$

has a non-trivial entire solution.

Some of the properties of these solutions are described in [3].

The case where the right side of (20) is rational in $w$ has been split into several different subcases, which have been treated by Kimura [78], Hirai [63] and Yanagihara [129]. The statement of the following theorem is due to Yanagihara [129].

Theorem 3.3 [63, 78, 129]. For any non-constant rational function $R(w)$, the difference equation

$$
w(z+1)=R(w)
$$

has a non-trivial meromorphic solution.

Some of the properties of these solutions are described in [2].

We outline here a straightforward proof of the existence of meromorphic solutions of (21) based on the methods used in [78, 121, 129] and on the Banach fixed point theorem. Full details of this approach can be found in [49].

In the Riccati or linear case $\operatorname{deg}_{w}(R)=1$ equation (21) is explicitly solvable in terms of exponential functions, see, for instance [82]. If $\operatorname{deg}_{w}(R) \geqslant 2$, then by [75] $R$ has a fixed point $\gamma$ such that either

$$
\left|R^{\prime}(\gamma)\right|>1
$$

or

$$
R^{\prime}(\gamma)=1
$$

In the case (22) equation (21) can be mapped into a Schröder functional equation, which is known to have meromorphic solutions (see, e.g., [121, 129]). We choose a slightly different approach which is based on a direct application of the Banach fixed point theorem. We will now show that (21) has a unique meromorphic solution in the complex plane such that

$$
(w(z)-\gamma) \lambda^{-z} \longrightarrow \alpha, \quad \text { as } \operatorname{Re}(z) \longrightarrow-\infty
$$

where $\left|R^{\prime}(\gamma)\right|=: \lambda>1$ and $\alpha \in \mathbb{C}$.

Expanding the right side of (21) as a Maclaurin series, and applying the transformation $w(z) \rightarrow \lambda^{z} w(z)+\gamma$ takes equation (21) into the form

$$
w(z+1)-w(z)=\sum_{j=2}^{\infty} \frac{1}{j !} R^{(j)}(\gamma) \lambda^{(j-1) z-1} w(z)^{j},
$$

which implies

$$
w(z)-w(z-m)=\sum_{j=2}^{\infty} \sum_{k=1}^{m} \frac{1}{j !} R^{(j)}(\gamma) \lambda^{(j-1)(z-k)-1} w(z-k)^{j} .
$$

Letting $m \longrightarrow \infty$, assumption (24) yields the formal identity

$$
w(z)=\alpha+\sum_{j=2}^{\infty} \sum_{k=1}^{\infty} \frac{1}{j !} R^{(j)}(\gamma) \lambda^{(j-1)(z-k)-1} w(z-k)^{j},
$$


which suggests to define an operator $T$ by

$$
T[w](z)=\alpha+\sum_{j=2}^{\infty} \sum_{k=1}^{\infty} \frac{1}{j !} R^{(j)}(\gamma) \lambda^{(j-1)(z-k)-1} w(z-k)^{j} .
$$

Let $X$ be the set of all functions $g(z)$, analytic and bounded in

$$
D(s, t)=\{z: \operatorname{Re}(z)<-s, \operatorname{Im}(z)>-t, t>0\}
$$

for which $g(z) \longrightarrow \alpha$ as $\operatorname{Re}(z) \longrightarrow-\infty$ and $\|g-\alpha\| \leqslant b$, where

$$
\|g-\alpha\|=\sup _{z \in D(s, t)}|g(z)-\alpha| .
$$

Then, choosing $s$ sufficiently large, $T$ is a contraction mapping in the Banach space $X$. Banach's fixed point theorem implies that $T$ has a unique fixed point $g(z)$ in $X$. This fixed point is the sought after solution of (21) satisfying (24), analytic in $D(s, t)$. By analytic continuation the solution $g(z)$ is meromorphic in $D(+\infty, t)$. Since $t$ is arbitrary, $g(z)$ is in fact meromorphic in the whole complex plane.

The case (23) can be found in a paper due to Kimura [78]. He uses a fixed point theorem due to Hukuhara based on the theory of normal families $[22,120]$ to study a general problem related to the iteration of analytic functions. The existence of meromorphic solutions of (21) in case (23) follows as a special case from his results. Since we are only concerned with the existence of meromorphic solutions of difference equations, for our purposes it is sufficient to apply the Banach fixed point theorem in case (23). We will show that if $\alpha \in \mathbb{C}, \delta \in(0,1)$ and $m \in \mathbb{N}$ is the smallest number such that $R^{(m+1)}(\gamma) \neq 0$, then there exists a constant $s>0$, and a unique solution $w(z)$ of (21), meromorphic in the complex plane, such that for all $z \in D(s):=\{z: \operatorname{Re}(z)<-s\}$,

$$
w(z)=\gamma-\frac{C_{1}}{z+\alpha+\beta \log z+W(z)}
$$

if $m=1$, and

$$
w(z)=\gamma-\frac{C_{m}}{\left(z+\alpha+\beta z^{\frac{m-1}{m}}+W(z)\right)^{\frac{1}{m}}},
$$

if $m \geqslant 2$. Here $\beta$ is a fixed constant,

$$
C_{m}=\left(\frac{m}{(m+1) !} R^{(m+1)}(\gamma)\right)^{-\frac{1}{m}}
$$

for $m \in \mathbb{N}$, and $|W(z)| \leqslant|z|^{-\frac{1}{m}+\delta}$ for all $z \in D(s)$.

By expanding the right side of (21) as a Taylor series and by substituting $w(z) \rightarrow$ $1 / w(z)+\gamma$, we obtain

$w(z+1)=\frac{w(z)}{1+\sum_{j=2}^{\infty} \frac{1}{j !} R^{(j)}(\gamma) w(z)^{-j+1}}=w(z)\left(1-\frac{1}{2} R^{\prime \prime}(\gamma) w(z)^{-1}+\cdots\right)$.

From now on we assume that $R^{\prime \prime}(\gamma) \neq 0$. The details in the case $R^{\prime \prime}(\gamma)=0$ can be found in [49]. By denoting $h(z)=-2 w(z) / R^{\prime \prime}(\gamma)$ equation (30) takes the form

$$
h(z+1)=h(z)+1+\sum_{j=1}^{\infty} c_{j} h(z)^{-j}
$$

where $c_{j} \in \mathbb{C}$ for all $j \in \mathbb{N}$. In summary, we have transformed equation (21) into

$$
w(z+1)=F(w(z)),
$$


where

$$
F(z)=z+1+\sum_{j=1}^{\infty} c_{j} z^{-j}
$$

Let $X$ be the family of analytic functions $W(z)$ such that

$$
|W(z)| \leqslant|z|^{-1+\delta}
$$

for all $z \in D(s)$. Then $X$ is a Banach space with respect to the sup-norm. By a straightforward calculation it can be seen that the operator

$$
\begin{aligned}
T[W](z)=\sum_{k=1}^{\infty} & \frac{c_{1}}{z-k} \sum_{n=1}^{\infty}\left(-\frac{\alpha+\beta \log (z-k)+W(z-k)}{z-k}\right)^{n} \\
& +\sum_{k=1}^{\infty} \sum_{j=2}^{\infty} \frac{c_{j}}{(z-k+\alpha+\beta \log (z-k)+W(z-k))^{j}} \\
& +\beta \sum_{k=1}^{\infty} \sum_{j=2}^{\infty}(-1)^{j} \frac{1}{j(z-k)^{j}},
\end{aligned}
$$

where $\beta=c_{1}$, is a contraction mapping satisfying

$$
|T[W](z)| \leqslant|z|^{-1+\delta}
$$

for all $z \in D(s)$. Therefore, the right side of (34) is absolutely and uniformly convergent, and hence $T[W](z)$ is analytic in $D(s)$. Thus, by Banach's fixed point theorem, the mapping $T: X \longrightarrow X$ has a unique fixed point. This is equivalent with the fact that

$$
Y(z)=z+\alpha+\beta \log z+W(z)
$$

is a solution of (31), by our choice of $T[W]$. The final step is to continue the analytic solution $Y(z)$ into a meromorphic solution in the whole complex plane by using equation (21).

\subsection{The QRT map}

Quispel, Roberts and Thompson have introduced a general system of second-order mappings which is explicitly integrable in terms of elliptic functions [105, 106]. Many discrete Painlevé equations have been first derived by implementing a discrete integrability criterion to a nonautonomous form of a mapping within the QRT family [109]. The QRT map is a generalization of the McMillan map [86].

The QRT family is defined by the system of two equations

$$
\begin{aligned}
x_{n+1} & =\frac{f_{1}\left(y_{n}\right)-x_{n} f_{2}\left(y_{n}\right)}{f_{2}\left(y_{n}\right)-x_{n} f_{3}\left(y_{n}\right)} \\
y_{n+1} & =\frac{g_{1}\left(x_{n+1}\right)-y_{n} g_{2}\left(x_{n+1}\right)}{g_{2}\left(x_{n+1}\right)-y_{n} g_{3}\left(x_{n+1}\right)}
\end{aligned}
$$

where

$$
\begin{aligned}
& \left(\begin{array}{l}
f_{1}(x) \\
f_{2}(x) \\
f_{3}(x)
\end{array}\right)=\left(\begin{array}{lll}
\alpha_{0} & \beta_{0} & \gamma_{0} \\
\delta_{0} & \varepsilon_{0} & \zeta_{0} \\
\kappa_{0} & \lambda_{0} & \mu_{0}
\end{array}\right)\left(\begin{array}{l}
x^{2} \\
x \\
1
\end{array}\right) \times\left(\begin{array}{lll}
\alpha_{1} & \beta_{1} & \gamma_{1} \\
\delta_{1} & \varepsilon_{1} & \zeta_{1} \\
\kappa_{1} & \lambda_{1} & \mu_{1}
\end{array}\right)\left(\begin{array}{l}
x^{2} \\
x \\
1
\end{array}\right) \\
& \left(\begin{array}{l}
g_{1}(x) \\
g_{2}(x) \\
g_{3}(x)
\end{array}\right)=\left(\begin{array}{lll}
\alpha_{0} & \delta_{0} & \kappa_{0} \\
\beta_{0} & \varepsilon_{0} & \lambda_{0} \\
\gamma_{0} & \zeta_{0} & \mu_{0}
\end{array}\right)\left(\begin{array}{l}
x^{2} \\
x \\
1
\end{array}\right) \times\left(\begin{array}{lll}
\alpha_{1} & \delta_{1} & \kappa_{1} \\
\beta_{1} & \varepsilon_{1} & \lambda_{1} \\
\gamma_{1} & \zeta_{1} & \mu_{1}
\end{array}\right)\left(\begin{array}{l}
x^{2} \\
x \\
1
\end{array}\right)
\end{aligned}
$$


and the coefficient matrices are constants. In the symmetric case

$$
\left(\begin{array}{ccc}
\alpha_{i} & \beta_{i} & \gamma_{i} \\
\delta_{i} & \varepsilon_{i} & \zeta_{i} \\
\kappa_{i} & \lambda_{i} & \mu_{i}
\end{array}\right)=\left(\begin{array}{ccc}
\alpha_{i} & \delta_{i} & \kappa_{i} \\
\beta_{i} & \varepsilon_{i} & \lambda_{i} \\
\gamma_{i} & \zeta_{i} & \mu_{i}
\end{array}\right), \quad i=0,1,
$$

the QRT family reduces into a single equation

$$
w_{n+1}=\frac{f_{1}\left(w_{n}\right)-w_{n-1} f_{2}\left(w_{n}\right)}{f_{2}\left(w_{n}\right)-w_{n-1} f_{3}\left(w_{n}\right)}
$$

by taking $x_{n}=w_{2 n}$ and $y_{n}=w_{2 n+1}$ in (36).

We will now follow a well-known procedure, see e.g. [6, 107], to show that the symmetric QRT difference equation

$$
\bar{w}=\frac{f_{1}(w)-\underline{w} f_{2}(w)}{f_{2}(w)-\underline{w} f_{3}(w)},
$$

related to the discrete integrable equation (37), possesses generically a two-parameter family of finite-order meromorphic solutions. (Here we have suppressed the $z$-dependence of $w(z)$ by writing $w \equiv w(z), \bar{w} \equiv w(z+1)$ and $\underline{w} \equiv w(z-1)$.) For the integration of the full asymmetric system (36) we refer to $[64,107]$. Equation (38) can be integrated to obtain

$$
\begin{aligned}
\bar{w}^{2} w^{2}+\left(\beta_{0}+\beta_{1} C_{1}\right)\left(\bar{w}^{2} w+\bar{w} w^{2}\right)+\left(\gamma_{0}+\gamma_{1} C_{1}\right)\left(\bar{w}^{2}+w^{2}\right) \\
+\left(\zeta_{0}+\zeta_{1} C_{1}\right)(\bar{w}+w)+\left(\varepsilon_{0}+\varepsilon_{1} C_{1}\right) \bar{w} w+\left(\mu_{0}+\mu_{1} C_{1}\right)=0,
\end{aligned}
$$

where $C_{1}$ is an arbitrary periodic function chosen here to be constant. Apart from certain degenerate special cases, the Möbius transformation

$$
w \longrightarrow \frac{a w+b}{c w+d},
$$

where $a, b, c, d$ are suitable constants, takes equation (39) into the form

$$
\bar{w}^{2} w^{2}+A\left(\bar{w}^{2}+w^{2}\right)+2 B \bar{w} w+1=0,
$$

where the constants $A$ and $B$ depend on $a, b, c, d$ and $C_{1}$, and on the coefficients of (39). From now on we assume that neither of the coefficients $A$ or $B$ vanish concentrating thus only on the generic case. Keeping this in mind we define the parameters $k$ and $\eta$ by the equations

$$
A=-\frac{1}{k \operatorname{sn}^{2} \eta},
$$

and

$$
B=\frac{\operatorname{cn} \eta \operatorname{dn} \eta}{k \operatorname{sn}^{2} \eta} .
$$

These choices of $A$ and $B$ imply that

$$
k+k^{-1}=\left(B^{2}-A^{2}-1\right) A^{-1} .
$$

Therefore, considering equation (40) as a quadratic equation for $\bar{w}$, and using the transformation

$$
w=k^{\frac{1}{2}} \operatorname{sn} u,
$$

where sn $u$ denotes the Jacobian elliptic sn function with argument $u$ and modulus $k$, we have

$$
\operatorname{sn} \bar{u}=\frac{\operatorname{cn} \eta \operatorname{dn} \eta \operatorname{sn} u \pm \operatorname{sn} \eta \operatorname{cn} u \operatorname{dn} u}{1-k^{2} \operatorname{sn}^{2} \eta \operatorname{sn}^{2} u} .
$$


This is solved by $u=\eta z+C_{2}$, where $C_{2} \in \mathbb{C}$ is a free parameter. Summarizing the above reasoning,

$$
w=\frac{a k^{\frac{1}{2}} \operatorname{sn}\left(\eta z+C_{2}\right)+b}{c k^{\frac{1}{2}} \operatorname{sn}\left(\eta z+C_{2}\right)+d}
$$

is a meromorphic solution of (38), where $C_{2} \in \mathbb{C}$, and $a, b, c, d, \eta$ and $k$ depend on the coefficients of (38) and on the free parameter $C_{1} \in \mathbb{C}$.

\subsection{Nonlinear higher order difference equations}

We conclude this section with an existence theorem due to Yanagihara on a class of higher order difference equations.

Theorem 3.4 (Yanagihara [131]). For any rational function

$$
R(w)=\frac{a_{p} w^{p}+\cdots+a_{0}}{b_{q} w^{q}+\cdots+b_{0}},
$$

where $a_{p}, \ldots, a_{0} \in \mathbb{C}, b_{q}, \ldots, b_{0} \in \mathbb{C}$ and $p \geqslant q+2$, the difference equation

$$
\alpha_{n} w(z+n)+\cdots+\alpha_{1} w(z+1)=R(w), \quad \alpha_{n}, \ldots, \alpha_{1} \in \mathbb{C},
$$

has a non-trivial meromorphic solution.

The method of proof of theorem 3.4 is similar to that of theorem 3.3. A fixed point theorem is first applied to find an analytic solution of (42) in a suitable sector, which is then continued to a meromorphic solution in the whole complex plane. Although this method works for large classes of difference equations, all meromorphic solutions found with it are free from poles in large parts of the complex plane. Therefore the method cannot be applied to equations solvable, for instance, in terms of elliptic functions, such as the QRT system in section 3.3 .

\section{Nevanlinna theory}

We have seen that large classes of difference equations admit meromorphic solutions. Therefore the existence of meromorphic solutions alone is not a good indicator of integrability. In order to characterize well-behaved meromorphic solutions we turn to Nevanlinna theory [92].

Nevanlinna theory has applications and analogies in many different fields of mathematics, such as differential equations [27, 34, 62, 40, 79], difference equations [121, 128, 129] number theory [98, 99, 115, 127], Brownian motion [18] and even mathematical logic [60]. Recently, there has been increasing interest in applying Nevanlinna theory to study meromorphic solutions of complex difference equations [20, 21, 48, 58, 67, 80], and in particular, to detect integrability in discrete equations [1, 49, 50, 111].

In what follows we will only give a brief outline of classical Nevanlinna theory without presenting proofs of the theorems. A more comprehensive description of Nevanlinna theory can be found, for example, in [19, 34, 54, 72]. For excellent historical accounts on the development of the theory we refer to [57, 81]. 


\subsection{Basic theory}

Picard's classical theorem states that a non-constant entire function can only omit one value in the complex plane [102]. This result was generalized by Borel who was able to go much deeper in the study of value distribution of entire functions [16]. He considered entire functions with finite order of growth

$$
\sigma(f):=\limsup _{r \rightarrow \infty} \frac{\log \log M(r, f)}{\log r},
$$

where $M(r, f):=\max _{|z|=r}|f(z)|$ is the maximum modulus of $f(z)$ in a disc of radius $r$. Borel showed that the number of $a$-points, i.e. the number of roots of the equation $f(z)=a$, of a non-constant entire function of finite order is determined by the rate of growth of the function, with at most one exceptional value $a$. Borel's result was later extended to infinite order functions by Blumenthal [15].

Picard's theorem immediately generalizes to the meromorphic case. Namely, if $f(z)$ is a meromorphic function omitting three distinct values $a, b$ and $c$, then $1 /(f(z)-a)$ is an entire function omitting $1 /(b-a)$ and $1 /(c-a)$, which implies that $f(z)$ must be a constant. Generalizing Borel's theorem to meromorphic functions is much more complicated. For one thing, it is not even immediately obvious how the notion of order should be defined for meromorphic functions. The first satisfactory extension of Borel's result to meromorphic functions was given by Nevanlinna [92]. He approached the problem by associating to each meromorphic function $f(z)$ three real-valued functions $m(r, f), N(r, f)$ and $T(r, f)$ which could be used indirectly to obtain information on the growth and value distribution of the meromorphic function $f(z)$ more efficiently than what had so far been achieved by a direct analysis of the meromorphic function itself.

The proximity function $m(r, f)$ is defined as the integral

$$
m(r, f):=\frac{1}{2 \pi} \int_{0}^{2 \pi} \log ^{+}\left|f\left(r \mathrm{e}^{\mathrm{i} \theta}\right)\right| \mathrm{d} \theta,
$$

where $\log ^{+} x:=\max \{0, \log x\}$ for all $x>0$. The proximity function describes, roughly speaking, how close on the average the values of $f(z)$ are to infinity on the circle $|z|=r$. Similarly, the growth of the function $m(r, a):=m\left(r, \frac{1}{f-a}\right)$ correlates with the proximity of the values of $f(z)$ to the value $a \in \mathbb{C}$ : the closer the values of $f(z)$ are to $a$ on the circle $|z|=r$, the larger the function $m(r, a)$ is.

The counting function $N(r, f)$ is an integral of the number of poles of $f(z)$ inside the disc $|z| \leqslant r$ with respect to the logarithmic measure $\mathrm{d} r / r$. It is sometimes also referred to as the integrated counting function, and it is defined as

$$
N(r, f):=\int_{0}^{r} \frac{n(t, f)-n(0, f)}{t} \mathrm{~d} t+n(0, f) \log r,
$$

where $n(r, f)$ is the number of poles of $f(z)$, counting multiplicities, in the disc $|z| \leqslant r$. Similarly, $N(r, a):=N\left(r, \frac{1}{f-a}\right)$ counts the number of $a$-points of $f(z)$ (i.e. the number of points $z \in \mathbb{C}$ such that $f(z)=a$ ) in the disc of radius $r$ centred at the origin.

The sum of the proximity function and the counting function is denoted by

$$
T(r, f):=m(r, f)+N(r, f)
$$

and it is called as the characteristic function of $f(z)$. The growth of the function $T(r, f)$ is a good measure of the complexity of the meromorphic function $f(z)$. The Nevanlinna characteristic is a convex increasing function of $\log r$, and differentiable almost everywhere. Moreover, by defining the order of growth of a meromorphic function $f(z)$ as

$$
\rho(f):=\limsup _{r \rightarrow \infty} \frac{\log T(r, f)}{\log r},
$$


it follows that $\rho(f)=\sigma(f)$ whenever $f(z)$ in entire [54]. Another remarkable property which the characteristic function possesses is known as the first main theorem of Nevanlinna theory,

$$
T(r, f)=T\left(r, \frac{1}{f-a}\right)+O(1) .
$$

The first main theorem implies that if $f(z)$ takes a value $a \in \mathbb{C} \cup\{\infty\}$ fewer times than average, i.e., the counting function $N(r, a)$ is relatively small, then the proximity function $m(r, a)$ must be large, and vice versa. Loosely speaking, if a meromorphic function assumes a certain value $a$ relatively few times, the values of $f(z)$ are 'near' the value $a$ in a large part of the complex plane.

The second main theorem of Nevanlinna theory extends Borel's result on the value distribution of entire functions to meromorphic functions. The exact statement and further discussion may be found in section 7.2 below.

\subsection{Exceptional sets}

In Nevanlinna theory one cannot usually avoid dealing with equations and inequalities which hold for 'most' values in the positive real axis. The 'small' set in which the desired property is not valid is called an exceptional set. When talking about an exceptional set one must always specify how large the set in question is. For example, if a set $E \subset[0, \infty)$ satisfies $\int_{E} \mathrm{~d} t<\infty$ it is said that $E$ has finite linear measure.

In this review we mostly end up dealing with slightly larger exceptional sets which may be of infinite linear measure, but have finite logarithmic measure $\int_{E} \frac{1}{t} \mathrm{~d} t<\infty$. For instance, if the characteristic function of a meromorphic function, say $g(z)$, satisfies $T(r, g)=o(T(r, f))$ outside of an exceptional set of $r$-values with finite logarithmic measure then it is said that $g(z)$ is small compared to $f(z)$, and the notation

$$
T(r, g)=S(r, f)
$$

is used. Intuitively speaking (45) just means that $T(r, g)$ is very small compared to $T(r, f)$ for most $r \geqslant 0$. We also denote by $\mathcal{S}(f)$ the family of all meromorphic functions which are small compared to $f(z)$.

\subsection{Useful properties of the characteristic function $T(r, f)$}

The characteristic function $T(r, f)$ possesses a number of useful properties which make it a relatively easy function to work with. As we already pointed out, the characteristic function is a convex increasing function of $\log r$, differentiable with respect to $r$ almost everywhere. In addition to this, the characteristic function provides a very convenient way to characterize rational functions. Namely, a meromorphic function $g(z)$ is rational if and only if $T(r, g)=O(\log r)$. This property is particularly handy when one is analysing the growth of non-rational meromorphic solutions of differential and difference equations with rational coefficients: the growth of the slower growing rational coefficients is relatively easy to distinguish from the growth of the fast growing meromorphic solution.

In addition to the first and second main theorems of Nevanlinna theory, there are a number of identities and inequalities for the characteristic function, counting function and the proximity function which are used frequently in Nevanlinna theory. Let $f(z), g(z)$ and $h(z)$ be meromorphic functions, and let $n \in \mathbb{N}$. Then

$$
T(r, f+g) \leqslant T(r, f)+T(r, g)+O(1)
$$




$$
\begin{aligned}
& T(r, f g) \leqslant T(r, f)+T(r, g) \\
& T\left(r, f^{n}\right)=n T(r, f) \\
& T(r, f g+g h+h f) \leqslant T(r, f)+T(r, g)+T(r, h)+O(1) \\
& T(r+1, f)=T(r, f)+S(r, f) \quad \text { if } f \text { is of finite order. }
\end{aligned}
$$

Relations (46)-(49) hold also for the counting function $N(r, f)$ and the proximity function $m(r, f)$, while the inequality (50) is true for the counting function but not necessarily for the proximity function. For a proof of the relations (46)-(48) see any standard reference in the Nevanlinna theory, for instance [54], for (49) see [111], and for (50) see [50]. In addition to (46)-(50) the identity

$$
m\left(r, \frac{f^{\prime}}{f}\right)=S(r, f)
$$

is very important in Nevanlinna theory. Relation (51) is called the lemma on the logarithmic derivative and it is an essential part of the proof of the second main theorem (110).

Finally, an identity originally due to Valiron [124] and generalized by Mohon'ko [89], has proved to be an extremely useful tool in the study of meromorphic solutions of differential, difference and functional equations. If $R(z, f)$ is a rational function of $f(z)$ with meromorphic coefficients $a_{\lambda}$ such that $T\left(r, a_{\lambda}\right)=S(r, f)$ for all $\lambda$, then

$$
T(r, R(z, f))=\operatorname{deg}_{f}(R(z, f)) T(r, f)+S(r, f) .
$$

In particular, if all coefficients of $R(z, f)$ are rational functions and $f(z)$ is non-rational, then $S(r, f)$ may be replaced with an error term of the growth $O(\log r)$ without an exceptional set.

Let us demonstrate the power of Nevanlinna theory in the analysis of meromorphic solutions of differential equations with a concrete example. Let $w(z)$ be a non-rational meromorphic solution of the differential equation

$$
w^{\prime}=P(z, w),
$$

where $P(z, w)$ is a polynomial in $w(z)$ with rational coefficients. Then, by (52), we have

$$
T\left(r, w^{\prime}\right)=\operatorname{deg}_{w}(P(z, w)) T(r, w)+O(\log r) .
$$

By the lemma on the logarithmic derivative (51) and (47), we obtain

$$
T\left(r, w^{\prime}\right) \leqslant N\left(r, w^{\prime}\right)+m(r, w)+m\left(r, \frac{w^{\prime}}{w}\right) \leqslant 2 T(r, w)+O(\log r) .
$$

Now, since $w(z)$ is non-rational $T(r, w) \neq O(\log r)$, and so by combining (54) and (55) we have $\operatorname{deg}_{w}(P(z, w)) \leqslant 2$. We conclude that if equation (53) has at least one non-rational meromorphic solution, then (53) must reduce to a Riccati differential equation

$$
w^{\prime}=a_{2}(z) w^{2}+a_{1}(z) w+a_{0}(z)
$$

with rational coefficients. We have just reproduced Yosida's proof [132] of the classical Malmquist theorem [85] in a special case. The treatment of the full rational case by using Nevanlinna theory is not much more complicated, see for example [79]. See also [27].

\section{Difference equations admitting finite-order meromorphic solutions}

In this section we explore the existence of sufficiently many solutions as a detector of integrability. 


\subsection{Difference equations of Painlevé type}

Yanagihara studied meromorphic solutions of nonlinear difference equations by using methods from Nevanlinna theory [129]. The following theorem can be understood as a difference analogue of Malmquist's theorem.

Theorem 5.1 (Yanagihara [129]). If the first-order difference equation

$$
\bar{w}=R(z, w),
$$

where $R(z, w)$ is rational in both arguments, admits a non-rational meromorphic solution of finite order, then $\operatorname{deg}_{w}(R)=1$.

Equation (56) with $\operatorname{deg}_{w}(R)=1$ is the difference Riccati equation,

$$
w(z+1)=\frac{\alpha(z) w(z)+\beta(z)}{\gamma(z) w(z)+\delta(z)}
$$

which possesses a continuum limit to the differential Riccati equation and is linearizable [110]. If $\gamma \not \equiv 0$ then equation (57) is linearized by the transformation

$$
w(z)=\frac{\alpha(z-1)}{\gamma(z-1)}\left[\frac{u(z)-u(z-1)}{u(z)}\right],
$$

where $u$ satisfies the second-order linear difference equation

$$
p(z) u(z+1)+q(z) u(z)+r(z) u(z-1)=0,
$$

where

$$
\begin{aligned}
& p(z)=\gamma(z-1)[\alpha(z) \delta(z)-\beta(z) \gamma(z)], \\
& q(z)=-\alpha(z)[\alpha(z-1) \gamma(z)+\gamma(z-1) \delta(z)], \\
& r(z)=\alpha(z-1) \alpha(z) \gamma(z) .
\end{aligned}
$$

Yanagihara's work has prompted a number of generalizations of theorem 5.1 to higher order difference equations $[1,58,80,111,130]$. We will now have a closer look at some of these results connected to difference Painlevé equations. Difference equations of the type

$$
\bar{w} \star \underline{w}=R(z, w),
$$

where $R(z, w)$ is rational in both of its arguments, and the operation $\star$ stands either for the addition or the multiplication, were studied in [1].

Theorem 5.2 (Ablowitz, Halburd and Herbst [1]). If the second-order difference equation (58) admits a non-rational meromorphic solution of finite order, then $\operatorname{deg}_{w}(R) \leqslant 2$.

The class of equations (58) with $\operatorname{deg}_{w}(R) \leqslant 2$ contains many equations considered to be of Painlevé type, including equations known as the difference Painlevé I-III. The following theorem is due to Ramani, Grammaticos, Tamizhmani and Tamizhmani [111].

Theorem 5.3 (Ramani, Grammaticos, Tamizhmani and Tamizhmani [111]). If the secondorder difference equation

$$
(\bar{w}+w)(w+\underline{w})=\frac{P(z, w)}{Q(z, w)},
$$

where $P(z, w)$ and $Q(z, w)$ are polynomials in $w$ having rational coefficients and no common roots, admits a non-rational meromorphic solution of finite order, then $\operatorname{deg}_{w}(P) \leqslant 4$ and $\operatorname{deg}_{w}(Q) \leqslant 2$. 
The class of equations singled out in theorem 5.3 contains the difference Painlevé IV equation, first derived by the singularity confinement method in [109]. Ramani et al have also given an analogue of theorem 5.3 which singles out a class of equations containing the difference Painlevé V equation [111].

The fact that many equations widely considered to be integrable lie within the classes of equations (58) with $\operatorname{deg}_{w}(R) \leqslant 2$, and (59) with $\operatorname{deg}_{w}(P) \leqslant 4$ and $\operatorname{deg}_{w}(Q) \leqslant 2$, supports the assertion that the existence of finite-order meromorphic solutions is a good detector of discrete integrable equations. On the other hand, many equations within these classes are generally considered to be non-integrable. In theorems 5.4 and 5.5 we have shown that equation (58) admits finite-order meromorphic solutions only in some very special cases which correspond to discrete equations considered to be integrable.

Instead of looking at equations with just rational coefficients, we have chosen a more general starting point, since some of the coefficients of discrete Painlevé equations are nonrational and we wish to single them out in their full generality. We say that a meromorphic solution $w$ of a difference equation is admissible if all coefficients of the equation are in $\mathcal{S}(w)$ (see [79] for admissible solutions in differential equations.) This means that the solution has faster growth than any of the coefficients in the sense of Nevanlinna theory (recall the exact definition from section 4.2.) For instance, all non-rational meromorphic solutions of an equation with rational coefficients are admissible. Note that theorems 5.2 and 5.3 remain true if 'rational' is replaced by 'meromorphic' and 'non-rational' is replaced by 'admissible'.

Theorem 5.4 [50]. If the equation

$$
\bar{w}+\underline{w}=R(z, w),
$$

where $R(z, w)$ is rational and irreducible in $w$ and meromorphic in $z$, has an admissible meromorphic solution of finite order, then either $w$ satisfies a difference Riccati equation

$$
\bar{w}=\frac{\bar{p} w+q}{w+p},
$$

where $p, q \in \mathcal{S}(w)$, or equation (60) can be transformed by a linear change in $w$ to one of the following equations:

$$
\begin{aligned}
& \bar{w}+w+\underline{w}=\frac{\pi_{1} z+\pi_{2}}{w}+\kappa_{1} \\
& \bar{w}-w+\underline{w}=\frac{\pi_{1} z+\pi_{2}}{w}+(-1)^{z} \kappa_{1} \\
& \bar{w}+\underline{w}=\frac{\pi_{1} z+\kappa_{1}}{w}+\frac{\pi_{2}}{w^{2}} \\
& \bar{w}+\underline{w}=\frac{\pi_{1} z+\pi_{3}}{w}+\pi_{2} \\
& \bar{w}+\underline{w}=\frac{\left(\pi_{1} z+\kappa_{1}\right) w+\pi_{2}}{(-1)^{-z}-w^{2}} \\
& \bar{w}+\underline{w}=\frac{\left(\pi_{1} z+\kappa_{1}\right) w+\pi_{2}}{1-w^{2}} \\
& \bar{w} w+w \underline{w}=p \\
& \bar{w}+\underline{w}=p w+q
\end{aligned}
$$

where $\pi_{k}, \kappa_{k} \in \mathcal{S}(w)$ are arbitrary finite-order periodic functions with period $k$. 
Equation (62) arises from the theory of orthogonal polynomials (see e.g. [83, 122]). It also appears in the matrix model approach to two-dimensional quantum gravity [12, 17, 32]. Historically, equation (62) is known as the discrete Painlevé I equation. The choice of name stems from the fact that a continuous limit, such as $w=-1 / 2+\varepsilon^{2} u, \kappa_{1}=-3, \pi_{1} z+\pi_{2}=$ $-\left(3+2 \varepsilon^{4} t\right) / 4, \varepsilon \rightarrow 0$, may be used to map (62) to the Painlevé I equation $u^{\prime \prime}=6 u^{2}+t$ [31]. In addition, equation (62) possesses a Lax pair, and it may be integrated by using isomonodromy techniques [32, 100]. Equation (64) is a known alternate difference Painlevé I equation [31, 37, 108]. It can also be mapped to the continuous Painlevé I equation by a suitable continuous limit, and its Lax pair has been given in [31]. Equation (65) is a known integrable equation with continuum limits to Painlevé I and IV, and its Lax pair has been given in $[36,39]$.

Equation (67) was found in connection with unitary matrix models of two-dimensional quantum gravity [101], and it was identified as the difference Painlevé II based on a continuum limit to the continuous Painlevé II equation. Equation (67) was also obtained as a similarity reduction of the discrete $\mathrm{mKdV}$ equation [94]. It possesses many special properties, including Lax pairs [74, 100], special Airy-type solutions [77, 119] and discrete Miura and auto-Bäcklund transformations [37].

Extensive studies of equations (62)-(65) and (67) suggest that they are all integrable [35]. In addition to possessing many properties indicative of integrability, including Lax pairs, they pass the singularity confinement test and have zero algebraic entropy [38, 61, 96]. They are also a part of the coalescence cascade for the discrete Painlevé equations [37]. Equation (66) is a slight variation of (67), and it appears to be related to an integrable alternating mapping introduced in [104]. Equation (61) is a difference Riccati equation, and (69) a linear difference equation. Equation (68) is linear in $w \underline{w}$ and possesses finite-order meromorphic solutions of many choices of $p$. Although some of the equations in theorem 5.4 can be transformed to each other, it is not clear whether such transformations preserve the admissibility of the solution $w$. It should also be noted that it is possible that two discrete equations can be transformed into each other while the corresponding difference equations cannot. This often comes down to the fact that, for $n \in \mathbb{Z}$ we have identities such as $\left((-1)^{n}\right)^{2}=1$, but for $z \in \mathbb{C},\left((-1)^{z}\right)^{2}=\exp (2 \pi i z)$. We conclude that the list of equations (61)-(69) contains all known integrable equations of the form (60) and apparently no non-integrable equations.

Instead of just repeating the proof of theorem 5.4 here, we take the opportunity to apply the method used to prove theorem 5.4 in [50] to single out the difference Painlevé III equation out of a natural class of difference equations. To this end, assume that $w$ is an admissible meromorphic solution of (58). By combining the identity (52) with the fact that

$$
T(r, w(z \pm 1)) \leqslant(1+\varepsilon) T(r+1, w)+O(1)
$$

holds for $\varepsilon>0$ when $r$ is sufficiently large [129] we obtain

$$
T(r+1, w) \geqslant \frac{\operatorname{deg}_{w}(R)}{2(1+\varepsilon)} T(r, w)+S(r, w) .
$$

Inequality (70) was obtained by following the reasoning in [1, 129], the only difference being that here the exceptional set associated with the error term $S(r, w)$ is bigger. If the degree of $R(z, w)$ with respect to $w$ is three or larger, then there exists an $\alpha<1$ such that

$$
T(r, w) \leqslant \alpha T(r+1, w)
$$

outside of a possible exceptional set $E$ with finite logarithmic measure. If $w$ is of finite order, then we arrive at a contradiction by combining (50) and (71). We conclude that if (58) has a finite-order meromorphic solution then $\operatorname{deg}_{w}(R) \leqslant 2$. 
In the following theorem we consider a natural subclass of $(58)$ with $\operatorname{deg}_{w}(R(z, w)) \leqslant 2$, and show that the existence of at least one finite-order meromorphic solution $w$ is sufficient to single out the difference Painlevé III equation from this subclass, provided that $w$ is not also an admissible solution of a difference Riccati equation. The full classification of (58) will be done elsewhere.

Theorem 5.5. Let $w$ be an admissible finite-order meromorphic solution of the equation

$$
\bar{w} \underline{w}=\frac{c_{2}\left(w-c_{+}\right)\left(w-c_{-}\right)}{\left(w-a_{+}\right)\left(w-a_{-}\right)}=: R(z, w)
$$

where the coefficients are meromorphic functions, $c_{2} \not \equiv 0$ and $\operatorname{deg}_{w}(R)=2$. If the order of the poles of $w$ is bounded, then either $w$ satisfies a difference Riccati equation

$$
\bar{w}=\frac{p w+q}{w+s},
$$

where $p, q, s \in \mathcal{S}(w)$, or equation (72) can be transformed by a bilinear change in $w$ to one of the equations

$$
\begin{aligned}
& \bar{w} \underline{w}=\frac{\underline{\gamma} w^{2}+\delta \lambda^{z} w+\gamma \mu \lambda^{2 z}}{(w-1)(w-\gamma)}, \\
& \bar{w} \underline{w}=\frac{w^{2}+\delta \mathrm{e}^{\mathrm{i} \pi z / 2} \lambda^{z} w+\mu \lambda^{2 z}}{w^{2}-1},
\end{aligned}
$$

where $\lambda \in \mathbb{C}$, and $\delta, \mu, \gamma \in \mathcal{S}(w)$ are arbitrary finite-order periodic functions such that $\delta$ and $\gamma$ have period 2 and $\mu$ has period 1 .

Equation (74) is the difference Painlevé III equation, first identified by the singularity confinement method [109]. It possesses a Lax pair [100], special discrete Riccati solutions [76] and Schlesinger transforms [73].

Compared to theorem 5.4, the assertion of theorem 5.5 contains an additional assumption concerning the orders of the poles of $w(z)$. This assumption can in fact be removed, and it is added here to avoid a number of technicalities which appear in the special case where the multiplicities of the poles of $w$ are allowed to grow without limit. In this case, for instance, it could happen that whenever $w$ has a pole, one of the coefficients of (72) also has a pole. Then, if the multiplicities of the poles of the coefficients remain small compared to the multiplicities of the poles of $w$, it is still possible that $w$ is admissible. The difficulties caused by this fact can be overcome, but presenting all the details here would not serve our purpose. Instead, we assume in what follows that the multiplicities of the poles of $w$ remain bounded and refer to [50] for a method of how to deal with sequences of poles where the multiplicities grow without limit.

\subsection{Tools from Nevanlinna theory}

The proof of theorem 5.4 in [50] and the proof of theorem 5.5 rely on some recent results on Nevanlinna theory for the shift operator $\Delta_{c} f:=f(z+c)-f(z)$. We state these results here before proceeding to prove theorem 5.5.

The lemma on the logarithmic derivative is the main ingredient in the proof of the second main theorem of Nevanlinna theory. It has also proved to be extremely useful in the analysis of value distribution of meromorphic solutions of differential equations [79]. The first result in this subsection is a difference analogue of the lemma on the logarithmic derivative and it is the foundation on which the Nevanlinna theory for the shift operator is built $[47,48]$. 
Chiang and Feng [20] obtained almost the same result as theorem 5.6 below, independently of [48], in a study concerning finite-order meromorphic solutions of linear difference equations.

Theorem 5.6 [47, 48]. Let $f(z)$ be a meromorphic function of finite order and let $c \in \mathbb{C}$. Then

$$
m\left(r, \frac{f(z+c)}{f(z)}\right)=S(r, f) .
$$

In this section $S(r, f)$ represents any function of $r$ which is $o(T(r, f))$ for all $r$ outside of an exceptional set with finite logarithmic measure. Stronger estimates of this error term for the theorems presented in this section are given in [47, 48].

Theorem 5.6 combined with standard methods from the Nevanlinna theory of differential equations is all that is needed to prove some rather powerful results on the value distribution of finite-order meromorphic solutions of large classes of nonlinear difference equations of arbitrarily high order. We state these results in their full generality first and then apply them to study finite-order meromorphic solutions of difference Painlevé equations.

Let $c_{j}, j=1, \ldots, n$, be a finite collection of complex numbers. Then a difference polynomial in $w(z)$ is a function which is polynomial in $w\left(z+c_{j}\right), j=1, \ldots, n$, with meromorphic coefficients $a_{\lambda}(z)$ such that $T\left(r, a_{\lambda}\right)=S(r, w)$ for all $\lambda$. The following theorem [48] is a difference analogue of the Clunie lemma [23].

Theorem 5.7 [48]. Let $w(z)$ be a non-constant finite-order meromorphic solution of

$$
w(z)^{n} P(z, w)=Q(z, w)
$$

where $P(z, w)$ and $Q(z, w)$ are difference polynomials in $w(z)$. If the degree of $Q(z, w)$ as a polynomial in $w(z)$ and its shifts is at most $n$, then

$$
m(r, P(z, w))=S(r, w) .
$$

In order to demonstrate exactly how theorem 5.7 can be used to obtain information about the density of poles of finite-order meromorphic solutions of difference equations, we consider as an example the following difference Painlevé equation

$$
\bar{w}+\underline{w}=\frac{\alpha z+\beta}{w}+\frac{\gamma}{w^{2}}
$$

with constant parameters $\alpha, \beta, \gamma$. Recall that we have suppressed the $z$-dependence by writing $w \equiv w(z), \bar{w} \equiv w(z+1)$ and $\underline{w} \equiv w(z-1)$. Suppose that $w$ is a non-rational meromorphic function of finite order. Then by considering (76) in the form

$$
w^{2}(\bar{w}+\underline{w})=(\alpha z+\beta) w+\gamma
$$

we may apply theorem 5.7 with $P(z, w)=\bar{w}+\underline{w}$ and $Q(z, w)=(\alpha z+\beta) w+\gamma$ thus obtaining

$$
m(r, \bar{w}+\underline{w})=S(r, w) .
$$

Since $T(r, \bar{w}+\underline{w})=2 T(r, w)+O(\log r)$ by (52) and (76), equation (77) yields

$$
N(r, \bar{w}+\underline{w})=2 T(r, w)+S(r, w) .
$$

Finally, since $N(r, \bar{w}+\underline{w}) \leqslant 2 N(r+1, w)=2 N(r, w)+S(r, w)$ (see (50) for the latter equality) we conclude by equation (78) that

$$
N(r, w)=T(r, w)+S(r, w) .
$$


In particular, this implies that all non-rational finite-order meromorphic solutions of the difference Painlevé equation (76) have infinitely many poles.

Just as theorem 5.7 can be used to study the pole distribution of finite-order meromorphic solutions of difference equations, the following theorem enables the analysis of the value distribution of solutions for finite values. It is an analogue of a result due to A Z Mohon'ko and V D Mohon'ko [90] on differential equations.

Theorem 5.8 [48]. Let $w(z)$ be a non-constant finite-order meromorphic solution of

$$
P(z, w)=0,
$$

where $P(z, w)$ is difference polynomial in $w(z)$. If $P(z, a) \not \equiv 0$ for a meromorphic function $a \in \mathcal{S}(w)$, then

$$
m\left(r, \frac{1}{w-a}\right)=S(r, w) .
$$

Consider again the difference Painlevé equation (76) as an example. If the parameter $\alpha$ is non-zero, then (76) does not have any constant solutions. Therefore theorem 5.8 yields

$$
m\left(r, \frac{1}{w-a}\right)=S(r, w)
$$

for all $a \in \mathbb{C}$. This immediately implies that all non-rational finite-order meromorphic solutions of the non-autonomous equation (76) have infinitely many $a$-points for all $a \in \mathbb{C}$.

\subsection{The proof of theorem 5.5}

Within the proof of theorem 5.5 we often say that there are at most $S(r, w)$ points $z_{0}$ with a certain property. By this we mean that the integrated counting function $N(r, \cdot)$ measuring the points with the property in question is at most of the growth $S(r, w)$. In loose terms this means that there are very few points with this property. Conversely, we use expressions like: 'There are more than $S(r, w)$ points such that...'. In precise terms, this means that

$$
\limsup _{r \rightarrow \infty} \frac{N(r, \cdot)}{T(r, w)}=c>0,
$$

where $c \in \mathbb{R}^{+} \cup\{\infty\}$, and $r$ runs to infinity in a set with infinite logarithmic measure. Intuitively speaking, this expression means that there exist a relatively large number of points with the property in question. For instance, by defining

$$
M:=\left\{\zeta: a_{ \pm}, c_{ \pm}, c_{2} \text { are analytic at } \zeta \text { and } c_{2}(\zeta) \neq 0\right\}
$$

we have that $\mathbb{C} \backslash M$ consists of at most $S(r, w)$ points.

Let $w$ be a meromorphic solution of (72) and choose a point $z_{0} \in M$ such that $w\left(z_{0}\right)=a_{ \pm}\left(z_{0}\right)$ with multiplicity $k$. Then by (72) $w\left(z_{0}+1\right)=\infty$ with multiplicity $l$ and $w\left(z_{0}-1\right)=\infty$ with multiplicity $k-l$ (and it may happen that either $k=0$ or $k-l=0$ which just means that either $w\left(z_{0}+1\right)$ or $w\left(z_{0}-1\right)$ is finite, respectively). We define the zero- $a_{ \pm}$ sequence of $w$ at $z_{0}$ to be the longest possible list of points $L\left(z_{0}, w\right)=\left(z_{-m}, \ldots, z_{0}, \ldots, z_{n}\right)$, where $z_{j}=z_{0}+j$, such that $w\left(z_{2 j}\right)=a_{ \pm}\left(z_{2 j}\right)$ at all $z_{2 j} \in L\left(z_{0}, w\right)$ and $w\left(z_{2 j+1}\right)=\infty$ at all $z_{2 j+1} \in L\left(z_{0}, w\right)$. The following lemma is an immediate consequence of equation (72) and the definition of $L\left(z_{0}, w\right)$.

Lemma 5.9. Let $w$ be a meromorphic solution of equation (72) such that $w\left(z_{0}\right)=a_{ \pm}\left(z_{0}\right), z_{0} \in$ $M$, with multiplicity $k$. Then either 
(a) $L\left(z_{0}, w\right)$ consists of at least three points, of which at least two are zeros of $w-a_{ \pm}$; or

(b) the total number of zeros of $w-a_{ \pm}$(counting multiplicities) in $L\left(z_{0}, w\right)$ divided by the total number of poles of $w$ is at most 1 .

When applying lemma 5.9 to study meromorphic solutions of (72) it is crucial to know that $w-a_{ \pm}$has sufficiently many zeros. Since (72) is irreducible, it is clear that none of the coefficients $a_{ \pm}, c_{ \pm}$is a solution of the equation (72). Therefore, by theorem 5.8, we have $m\left(r, \frac{1}{w-a_{ \pm}}\right)=S(r, w)$ and $m\left(r, \frac{1}{w-c_{ \pm}}\right)=S(r, w)$ which implies that $N\left(r, \frac{1}{w-c_{ \pm}}\right)=T(r, w)+S(r, w)$. Also, by applying theorem 5.7 with $P(z, w)=\bar{w} \underline{w}$ and $Q(z, w)=c_{2} w^{2}+c_{1} w+c_{0}-(a w+b) \bar{w} \underline{w}$, we obtain $m(r, \bar{w} \underline{w})=S(r, w)$. Therefore, by (47), (48) and theorem 5.6, we have

$$
m(r, w) \leqslant \frac{1}{2} m(r, \bar{w} \underline{w})+\frac{1}{2} m\left(r, \frac{w}{\bar{w}}\right)+\frac{1}{2} m\left(r, \frac{w}{w}\right)=S(r, w),
$$

which implies that $N(r, w)=T(r, w)+S(r, w)$. In other words, $w$ has a large number of poles, and $w-a_{ \pm}$and $w-c_{ \pm}$have a large number of zeros (for both choices of \pm ).

Clearly all zeros of $w-a_{ \pm}$(which are in $M$ ) are a part of some sequences $L\left(z_{0}, w\right)$. Let $N_{b}(r, w)$ be the integrated counting function for those poles of $w$ which are a part of a type (b) sequence defined in the statement of lemma 5.9. If there are more than $S(r, w)$ such poles, then by the definition of 'more than $S(r, w)$ ' there exists a constant $c>0$ such that

$$
N_{b}(r, w) \geqslant c T(r, w)
$$

for all $r$ in a set $E$ with infinite logarithmic measure. Hence, by (52), (72), (81) and lemma 5.9, we have

$$
\begin{aligned}
2 T(r, w)= & T(r, \bar{w} \underline{w})+S(r, w)=N(r, \bar{w} \underline{w})-N_{b}(r, \bar{w} \underline{w})+N_{b}(r, \bar{w} \underline{w})+S(r, w) \\
& \leqslant 2\left(N(r+1, w)-N_{b}(r+1, w)\right)+N_{b}(r+1, w)+S(r, w) \\
& \leqslant(2-c) T(r+1, w)+S(r, w)
\end{aligned}
$$

for all $r$ in a set with infinite logarithmic measure. Therefore $w$ is of infinite order by inequality (50).

We conclude that if $w$ is of finite order, then there are at least $T(r, w)+S(r, w)$ points $z_{0}$ such that $w\left(z_{0}+1\right)=\infty$ and one of the four following relations is valid:

$$
\begin{array}{llll}
w\left(z_{0}\right)=a_{-}\left(z_{0}\right) & \text { and } & & w\left(z_{0}+2\right)=a_{+}\left(z_{0}+2\right) \\
w\left(z_{0}\right)=a_{+}\left(z_{0}\right) & \text { and } & w\left(z_{0}+2\right)=a_{-}\left(z_{0}+2\right) \\
w\left(z_{0}\right)=a_{+}\left(z_{0}\right) & \text { and } & & w\left(z_{0}+2\right)=a_{+}\left(z_{0}+2\right) \\
w\left(z_{0}\right)=a_{-}\left(z_{0}\right) & \text { and } & & w\left(z_{0}+2\right)=a_{-}\left(z_{0}+2\right) .
\end{array}
$$

Since $N\left(r, \frac{1}{w-a_{+}}\right)=T(r, w)+S(r, w)$ holds true for both $a_{-}$and $a_{+}$, exactly one of the following four cases is valid:

(i) There are more than $S(r, w)$ points such that both (85) and (86) hold.

(ii) There are more than $S(r, w)$ points such that both (83) and (84) hold, and at most $S(r, w)$ points such that either (85) or (86) holds.

(iii) There are more than $S(r, w)$ points such that (84) holds, and at most $S(r, w)$ points such that (83), (85) or (86) holds.

(iv) There are more than $S(r, w)$ points such that (83) holds, and at most $S(r, w)$ points such that (84), (85) or (86) holds. 
The transformation $w \rightarrow 1 / w$ takes the equation (72) into the form

$$
\bar{w} \underline{w}=\frac{\frac{a_{+} a_{-}}{c_{2} c_{+} c_{-}}\left(w-1 / a_{+}\right)\left(w-1 / a_{-}\right)}{\left(w-1 / c_{+}\right)\left(w-1 / c_{-}\right)},
$$

which still of the form (72) but with different coefficients. Therefore there are at least $T(r, w)+S(r, w)$ points such that one of the following options is valid:

$$
\begin{array}{llll}
w\left(z_{0}\right)=1 / c_{-}\left(z_{0}\right) & \text { and } & & w\left(z_{0}+2\right)=1 / c_{+}\left(z_{0}+2\right) \\
w\left(z_{0}\right)=1 / c_{+}\left(z_{0}\right) & \text { and } & & w\left(z_{0}+2\right)=1 / c_{-}\left(z_{0}+2\right) \\
w\left(z_{0}\right)=1 / c_{+}\left(z_{0}\right) & \text { and } & w\left(z_{0}+2\right)=1 / c_{+}\left(z_{0}+2\right) \\
w\left(z_{0}\right)=1 / c_{-}\left(z_{0}\right) & \text { and } & w\left(z_{0}+2\right)=1 / c_{-}\left(z_{0}+2\right) .
\end{array}
$$

Thus exactly one of the following statements is valid simultaneously to one of the (i)-(iv):

(i) There are more than $S(r, w)$ points such that both (90) and (91) hold.

(ii) There are more than $S(r, w)$ points such that both (88) and (89) hold, and at most $S(r, w)$ points such that either (90) or (91) holds.

(iii) There are more than $S(r, w)$ points such that (89) holds, and at most $S(r, w)$ points such that (88), (90) or (91) holds.

(iv) There are more than $S(r, w)$ points such that (88) holds, and at most $S(r, w)$ points such that (89), (90) or (91) holds.

In what follows we shall see that the case in which both (ii) and (ii') are both valid leads to the full difference Painlevé III equation (74). Cases in which (i) and (i'), (i) and (ii') or (ii) and (i') hold true, equation (72) is either reduced into (75), or into a special case of the difference Painlevé III equation. In all cases in which any of (iii), (iii'), (iv) or (iv') is valid the finite-order meromorphic solution $w$ satisfies a Riccati difference equation (73).

5.3.1. The difference Painlevé III. We consider first the case in which (i) and (i') are valid. Since by assumption there are more than $S(r, w)$ points such that $(85)$ holds, it follows by equation (72) that there are more than $S(r, w)$ points $z_{0}$ such that $c_{2}\left(z_{0}\right)=$ $a_{+}\left(z_{0}-1\right) a_{+}\left(z_{0}+1\right)$, and so in fact $c_{2}(z) \equiv a_{+}(z-1) a_{+}(z+1)$ since $a_{ \pm} \in \mathcal{S}(w)$. Similarly, $c_{2}(z) \equiv a_{-}(z-1) a_{-}(z+1)$, and since $a_{+}$and $a_{-}$are of finite order, either $a_{+}(z) \equiv a_{-}(z)$ or $a_{+}(z) \equiv-a_{-}(z)$. Since the former equation is in contradiction with the assumptions of theorem 5.5 we have $a_{+}(z) \equiv-a_{-}(z)$. Similarly $c_{+}(z) \equiv-c_{-}(z)$, and so equation (72) can be written in the form

$$
\bar{w} \underline{w}=\frac{\frac{a_{+}^{2}}{\bar{a}_{+} \underline{a}_{+} c_{+}^{2}}\left(w^{2}-1 / a_{+}^{2}\right)}{\left(w^{2}-1 / c_{+}^{2}\right)} .
$$

By combining the assumption ( $\left.\mathrm{i}^{\prime}\right)$ and equation (92) it follows that

$$
\frac{\bar{a}_{+}}{\bar{c}_{+}} \cdot \frac{\underline{a}_{+}}{\underline{c}_{+}}=\frac{a_{+}^{2}}{c_{+}^{2}},
$$

which can be solved to obtain $a_{+} / c_{+}=\mu \lambda^{z}$, where $\mu$ is an arbitrary finite-order periodic function with period 1 , and $\lambda$ is any non-zero complex number. Since the transformation $w \rightarrow a_{+} w$ takes equation (72) into the form

$$
\bar{w} \underline{w}=\frac{w^{2}-c_{+}^{2} / a_{+}^{2}}{w^{2}-1}
$$


we conclude that if, in the case (i) and (i'), equation (72) has a finite-order meromorphic solution, then (72) is reduced to

$$
\bar{w} \underline{w}=\frac{w^{2}-\mu \lambda^{2 z}}{w^{2}-1}
$$

which is a special case of the difference Painlevé III equation.

Consider now the case (ii) and (ii'). Similarly as in the case (i), the assumptions of the case (ii) together with equation (72) imply

$$
c_{2}=\underline{a}_{+} \bar{a}_{-}=\underline{a}_{-} \bar{a}_{+} .
$$

Equation (95) may be solved to obtain that $a_{+} / a_{-}$is an arbitrary periodic function with period 2. Similarly, the transformed equation (87) with the assumption (ii') yields

$$
\frac{c_{2} c_{+} c_{-}}{a_{+} a_{-}}=\underline{c}_{+} \bar{c}_{-}=\underline{c}_{-} \bar{c}_{+}
$$

which can be combined with (95) to obtain

$$
\left(\frac{c_{+} c_{-}}{a_{+} a_{-}}\right)^{2}=\frac{\bar{c}_{+} \bar{c}_{-}}{\bar{a}_{+} \bar{a}_{-}} \frac{\underline{c}_{+} \underline{c}_{+}-\underline{a}_{-}}{\underline{a}_{-}} .
$$

On one hand, by solving equation (97) we have

$$
\frac{c_{+} c_{-}}{a_{+} a_{-}}=\mu \lambda^{2 z}
$$

where $\mu$ is an arbitrary finite-order periodic function with period 1 , and $\lambda$ is a complex constant. On the other hand, by assumption (ii'),

$$
\begin{aligned}
& w\left(z_{0}+1\right) w\left(z_{0}-1\right)=\frac{c_{2}\left(z_{0}\right) c_{+}\left(z_{0}\right) c_{-}\left(z_{0}\right)}{a_{+}\left(z_{0}\right) a_{-}\left(z_{0}\right)} \\
& w\left(z_{0}+1\right)^{2}-\left(c_{+}\left(z_{0}+1\right)+c_{-}\left(z_{0}+1\right)\right) w\left(z_{0}+1\right)+c_{+}\left(z_{0}+1\right) c_{-}\left(z_{0}+1\right)=0 \\
& w\left(z_{0}-1\right)^{2}-\left(c_{+}\left(z_{0}-1\right)+c_{-}\left(z_{0}-1\right)\right) w\left(z_{0}-1\right)+c_{+}\left(z_{0}-1\right) c_{-}\left(z_{0}-1\right)=0
\end{aligned}
$$

at more than $S(r, w)$ points $z_{0}$. By combining these equations and using (97), we obtain

$$
\begin{aligned}
\left(\left(c_{+}\left(z_{0}+1\right)+\right.\right. & \left.c_{-}\left(z_{0}+1\right)\right) \frac{c_{2}\left(z_{0}\right) c_{+}\left(z_{0}\right) c_{-}\left(z_{0}\right)}{a_{+}\left(z_{0}\right) a_{-}\left(z_{0}\right)} \\
& \left.-\left(c_{+}\left(z_{0}-1\right)+c_{-}\left(z_{0}-1\right)\right) c_{+}\left(z_{0}+1\right) c_{-}\left(z_{0}+1\right)\right) w\left(z_{0}-1\right)=0 .
\end{aligned}
$$

Since by assumption $w\left(z_{0}-1\right)=c_{ \pm}\left(z_{0}-1\right)$ and since there are more than $S(r, w)$ points $z_{0}$ such that (99) holds, the coefficient of $w$ in (99) must vanish identically. In addition, by using the fact that $c_{2}=\bar{a}_{+} \underline{a}_{-}$, we obtain the equation

$$
\frac{\underline{c}_{+}+\underline{c}_{-}}{\underline{a}_{-}} \cdot \frac{\bar{c}_{+} \bar{c}_{-}}{\bar{a}_{+} \bar{a}_{-}}=\frac{\bar{c}_{+}+\bar{c}_{-}}{\bar{a}_{-}} \cdot \frac{c_{+} c_{-}}{a_{+} a_{-}}
$$

which, by taking (98) into account, can be solved to have

$$
\frac{c_{+}+c_{-}}{a_{-}}=-\delta^{\prime} \lambda^{z}
$$

where $\delta^{\prime}$ is an arbitrary periodic function with period 2, and $\lambda$ is as in (98). Therefore equation (72) takes the form

$$
\bar{w} \underline{w}=\frac{\bar{a}_{+} \underline{a}_{-}\left(w^{2}+a_{-} \delta^{\prime} \lambda^{z} w+a_{+} a_{-} \mu \lambda^{2 z}\right)}{\left(w-a_{+}\right)\left(w-a_{-}\right)} .
$$


By denoting $\gamma:=a_{-} / a_{+}$and $\delta:=\gamma \delta^{\prime}$ (recall that $a_{-} / a_{+}$is periodic with period 2) and using the transformation $w \rightarrow a_{+} w$, we obtain

$$
\bar{w} \underline{w}=\frac{\gamma}{(w-1)(w-\gamma)}
$$

which is the difference Painlevé III equation (74).

Suppose now that (i) and (ii') are valid. Then we have already shown that $c_{2}=\bar{a}_{+} \underline{a}_{+}=$ $\bar{a}_{-} \underline{a}_{-}$and that $a_{+}=-a_{-}$. A similar calculation to that of above shows that (97) and (98) hold also in this case. However, instead of (100), we have the equation

$$
\frac{\underline{c}_{+}+\underline{c}_{-}}{\underline{a}_{-}} \cdot \frac{\bar{c}_{+} \bar{c}_{-}}{\bar{a}_{+} \bar{a}_{-}}=-\frac{\bar{c}_{+}+\bar{c}_{-}}{\bar{a}_{-}} \cdot \frac{c_{+} c_{-}}{a_{+} a_{-}}
$$

which has the solution

$$
\frac{c_{+}+c_{-}}{a_{-}}=-\delta^{\prime} \mathrm{e}^{\mathrm{i} \pi z / 2} \lambda^{z}
$$

rather than (101). Therefore in this case equation (72) has the form

$$
\bar{w} \underline{w}=\frac{w^{2}+\delta \mathrm{e}^{\mathrm{i} \pi z / 2} \lambda^{z} w+\mu \lambda^{2 z}}{w^{2}-1} .
$$

Consider finally the case (ii) and (i'). In this case (98) is still valid, and in addition we have $c_{+}=-c_{-}$. Hence equation (72) becomes

$$
\bar{w} \underline{w}=\frac{\underline{\gamma} w^{2}+\mu \lambda^{2 z}}{(w-1)(w-\gamma)}
$$

which is a special case of (74).

5.3.2. The difference Riccati equation. Since cases (iii), (iii'), (iv) and (iv') are very similar to each other, we are satisfied with presenting explicit details only in the case (iii). Note that it does not matter which of the cases $\left(\mathrm{i}^{\prime}\right)-\left(\mathrm{iv}^{\prime}\right)$ is valid simultaneously to (iii), we end up with $w$ satisfying a Riccati difference equation nevertheless.

We have already shown that all except at most $S(r, w)$ zeros of $w-a_{ \pm}$are in a sequence $L\left(z_{0}, w\right)$ containing at least two $a_{ \pm}$-points of $w$. From the statement (iii) it follows that those sequences must contain exactly one $a_{+}$-point and one $a_{-}$-point (otherwise there would be more than $S(r, w)$ points where one of the relations (83), (85) or (86) would be valid).

Although all $a_{ \pm}$-points of $w$ (which belong to the set $M$ ) are part of some sequence $L\left(z_{0}, w\right)$ there might be poles of $w$ which are not. However, if there are more than $S(r, w)$ poles which are not a part of any $L\left(z_{0}, w\right)$ then $w$ would be of infinite order by a similar calculation as in (82).

We have so far shown that all except at most $S(r, w)$ poles of $w$ are part of a sequence $L\left(z_{0}, w\right)=\left(l, a_{+}, \infty, a_{-}, m\right)$ where $l$ and $m$ are finite values. Suppose that the pole in the sequence $L\left(z_{0}, w\right)$ is of multiplicity $k$ and that either $w-a_{+}$or $w-a_{-}$has a zero of order strictly less than $k$ in $L\left(z_{0}, w\right)$ (this happens if either $l$ or $m$ is zero). In such a sequence the number of zeros of $w-a_{ \pm}$(counting multiplicities) divided by the number of poles of $w$ is strictly less than 2, say $\alpha$. If there are more than $S(r, w)$ poles in sequences of this type then, similarly as in (82), there is a $c>0$ such that

$$
2 T(r, w) \leqslant(2-(2-\alpha) c) T(r+1, w)+S(r, w) .
$$

Since $2-(2-\alpha) c<2$ inequality (102) combined with (50) yields a contradiction. Thus in all except at most $S(r, w)$ sequences $L\left(z_{0}, w\right)$ the multiplicities of all points are the same. Hence,

$$
U:=\left(w-a_{+}\right)\left(\bar{w}-\bar{a}_{-}\right)
$$


satisfies $N(r, U)=S(r, w)$. Also, $m(r, U)=S(r, w)$ by (81) and (103). We conclude that $T(r, U)=S(r, w)$ and so equation (103) is the Riccati difference equation (73).

\section{Linear difference equations}

It is well known that the linear first-order difference equation

$$
w(z+1)=a(z) w(z)
$$

is explicitly solvable in terms of exponential and gamma functions if the coefficient $a(z)$ is a rational function, see e.g. [82]. Whittaker [128] has shown that in the more general case where the coefficient $a(z)$ is a finite-order meromorphic function, equation (104) admits a meromorphic solution $w$ such that $\rho(a) \leqslant \rho(w) \leqslant \rho(a)+1$. Recently Chiang and Feng [20] showed that if $a(z)$ an entire function of finite order, then all solutions of (104) satisfy $\rho(w) \geqslant \rho(a)+1$.

The behaviour of meromorphic solutions of higher order linear difference equations remains relatively unknown. The first step towards understanding the growth of meromorphic solutions of linear difference equations of arbitrary order was given by Ishizaki and Yanagihara [67] who studied entire solutions of the equation

$$
a_{n}(z) \Delta^{n} w(z)+\cdots+a_{1}(z) \Delta w(z)+a_{0}(z) w(z)=0
$$

with polynomial coefficients. They showed that if an entire solution of (105) has relatively slow growth, then the order of the solution is a rational number expressible in terms of the degrees of the polynomial coefficients. The exact formulation of their result is as follows. The Newton polygon for (105) is defined as the convex hull of $\cup_{j=0}^{n}\{(x, y): x \geqslant j, y \leqslant$ $\left.\operatorname{deg} a_{n-j}(z)-(n-j)\right\}$.

Theorem 6.1 (Ishizaki and Yanagihara [67]). Let $w(z)$ be a non-rational entire solution of (105) and of order $\chi<1 / 2$. Then

$$
\log M(r, w)=L r^{\chi}(1+o(1)),
$$

where $\chi$ is a slope of the Newton polygon for equation (105), and $L>0$ is a constant. In particular, $\chi>0$.

Wiman-Valiron theory (see [56] for a review) is an efficient tool when studying the growth of entire solutions of differential equations. In order to prove theorem 6.1, Ishizaki and Yanagihara developed a difference analogue of the Wiman-Valiron method for slow-growing entire solutions of linear difference equations. They also give examples of entire solutions of (105) satisfying the conditions of theorem 6.1.

Another approach to study meromorphic solutions of linear difference equations by Chiang and Feng relies on logarithmic difference estimates of finite-order meromorphic functions [20]. This approach enabled Chiang and Feng to find a uniform lower bound for the order of growth of meromorphic solutions of large classes of linear difference equations.

Theorem 6.2 (Chiang and Feng [20]). Let $w(z)$ be a meromorphic solution of

$$
a_{n}(z) w(z+n)+\cdots+a_{1}(z) w(z+1)+a_{0}(z) w(z)=0
$$

where the coefficients $a_{n}(z), \ldots, a_{0}(z)$ are polynomials. If there exists an integer $m \in$ $\{0, \ldots, n\}$ such that

$$
\operatorname{deg}\left(a_{m}\right)>\max _{\substack{0 \leqslant j \leqslant n \\ j \neq m}}\left\{\operatorname{deg}\left(a_{j}\right)\right\}
$$

then $\rho(w) \geqslant 1$. 
Chiang and Feng also considered the case where the coefficients of (106) are entire functions of finite order satisfying a growth condition analogous to (107). In particular, they showed that if the growth of one of the coefficients dominates the growth of others, then the order of growth of all non-zero solutions is at least one higher than the order of the coefficient with maximal growth [20].

One of the key tools used in the proof of theorem 6.2 is the following pointwise logarithmic difference estimate, which is analogous to an estimate on the logarithmic derivative due to Gundersen [44].

Theorem 6.3 (Chiang and Feng [20]). Let $w(z)$ be a meromorphic function of finite order $\sigma$, and let $\varepsilon>0$. Then

$$
\exp \left(-r^{\sigma-1+\varepsilon}\right) \leqslant\left|\frac{w(z+1)}{w(z)}\right| \leqslant \exp \left(r^{\sigma-1+\varepsilon}\right)
$$

for all $r=|z|$ outside of an exceptional set with finite logarithmic measure.

Ruijsenaars has studied the first-order linear difference equation

$$
F(z+\mathrm{i} a / 2)=\Phi(z) F(z-\mathrm{i} a / 2),
$$

where $\Phi(z)$ is a meromorphic function satisfying certain regularity conditions, and $a>0$ is the step size of the equation. He found a new representation of solutions of (108) with no zeros and poles in a strip parallel to the real axis, and satisfying a certain growth condition in the same strip. Ruijsenaars named solutions of this type as 'minimal'. Special difference equations within the class (108) give rise to minimal solutions which can be understood as generalized gamma functions of hyperbolic, trigonometric and elliptic type, the standard gamma function being of rational type. These generalized gamma functions can be used to express the scattering and weight functions of certain integrable quantum systems [116].

The following theorem due to Chiang and Ruijsenaars [21] contains as special cases some of the Ruijsenaars' earlier results on minimal solutions [116, 117].

Theorem 6.4 (Chiang and Ruijsenaars [21]). Assume that $\psi(z)$ is a function that is analytic in $S_{c}:=\{z \in \mathbb{C}:|\operatorname{Im}(z)|<c\}$ for some $c>0$ and that satisfies

$$
\psi(z)=O\left(|z|^{\nu}\right), \quad z \in S_{c}, \quad|z| \rightarrow \infty,
$$

for some $v>-1$, uniformly in closed substrips of $S_{c}$. Then the function

$$
h(z):=\frac{\pi}{2 \mathrm{i} a^{2}} \int_{-\infty}^{\infty} \frac{\psi(z-x)}{\cosh (\pi x / a)} \mathrm{d} x, \quad z \in S_{c},
$$

admits analytic continuation to $S_{c+a / 2}$. Introducing

$$
H(z):=\int_{0}^{z} h(w) \mathrm{d} w, \quad z \in S_{c+a / 2},
$$

there exists $\gamma \in \mathbb{C}$ such that the function $\kappa(z):=H(z)+\gamma z$ satisfies the difference equation

$$
\kappa(z+\mathrm{i} a / 2)-\kappa(z-\mathrm{i} a / 2)=\psi(z), \quad z \in S_{c} .
$$

Moreover, we have the bound

$$
\kappa(z)-\gamma z=O\left(|z|^{v+1}\right), \quad z \in S_{c+a / 2}, \quad|z| \rightarrow \infty,
$$

uniformly in closed substrips of $S_{c+a / 2}$.

Minimal solutions of (108) can be obtained by applying an exponential transformation to equation (109). A large class of coefficients $\Phi(z)$ such that $\psi(z)=\log \Phi(z)$ (for a fixed 
branch of the logarithm) satisfies the conditions of theorem 6.4 can be constructed by using Hadamard products [21].

As we mentioned earlier, Whittaker has shown that if $\Phi(z)$ is of finite order, then there exists a meromorphic solution $F$ of $(108)$ such that $\rho(F) \leqslant \rho(\Phi)+1$. Whittaker's proof of the existence is non-constructive in the sense that the meromorphic solution $F(z)$ such that $\rho(F) \leqslant \rho(\Phi)+1$ is not expressed explicitly in terms of the coefficient $\Phi(z)$. Chiang and Ruijsenaars showed that if the coefficient of (108) is a meromorphic function of finite order $\rho$, and if equation (108) admits a special type of minimal solution satisfying an asymptotic growth condition depending on $\rho$, then the order the minimal solution is at most $\rho+1$ [21]. Their approach is constructive in the sense that the obtained minimal solution has an explicit representation in terms of the coefficient of (108).

\section{Value distribution of differences of meromorphic functions}

In this section we will present a number of recent results on the value distribution of shifts of finite-order meromorphic functions, which may prove to be important tools in the study of analytic difference equations in the future.

\subsection{Zeros of differences of meromorphic functions}

Bergweiler and Langley have shown that differences of relatively slow growing meromorphic functions behave asymptotically like their derivatives in large parts of the complex plane [11]. The following lemma is an example of their results of this type.

Lemma 7.1 (Bergweiler and Langley [11]). Let $n \in \mathbb{N}$. Let $f$ be non-rational and meromorphic of order less than 1 in the plane. Then

$$
\Delta^{n} f(z) \sim f^{(n)}(z) \quad \text { as } \quad z \rightarrow \infty \text { in } \mathbb{C} \backslash E_{n},
$$

where $E_{n} \subset \mathbb{C}$ is a countable union of discs

$$
E_{n}=\bigcup_{j=1}^{\infty} B\left(b_{j}, r_{j}\right) \quad \text { such that } \quad \lim _{j \rightarrow \infty}\left|b_{j}\right|=\infty \text { and } \quad \sum_{j=1}^{\infty} \frac{r_{j}}{\left|b_{j}\right|}<\infty .
$$

A combination of asymptotic relations between differences and derivatives, such as lemma 7.1, with methods used to study derivatives of meromorphic functions is a useful tool in the study of differences of meromorphic functions with slow growth. The proof of the following theorem, for instance, is based on lemma 7.1 and the standard Wiman-Valiron theory.

Theorem 7.2 (Bergweiler and Langley [11]). Let $n \in \mathbb{N}$. Let $f$ be a non-polynomial entire function of order $\rho<1 / 2$, and set

$$
G(z)=\frac{\Delta^{n} f(z)}{f(z)}
$$

If $G$ is non-rational then $G$ has infinitely many zeros. In particular if $f$ has order less than $\min \left\{\frac{1}{n}, \frac{1}{2}\right\}$ then $G$ is non-rational and has infinitely many zeros.

\subsection{Second main theorem of Nevanlinna theory}

We will now take a short digression from differences of meromorphic functions in order to complete the introduction to Nevanlinna theory started in sections 4 and 5.2. 
The second main theorem of Nevanlinna theory says that if $q \geqslant 3$ and $a_{1}, a_{2}, \ldots, a_{q}$ are distinct complex numbers, then

$$
(q-2) T(r, f) \leqslant \sum_{j=1}^{q} N\left(r, \frac{1}{f-a_{j}}\right)-N_{1}(r, f)+S(r, f),
$$

where $N_{1}(r, f):=2 N(r, f)-N\left(r, f^{\prime}\right)+N\left(r, \frac{1}{f^{\prime}}\right)$ is a positive quantity measuring the number of multiple $a$-points, and

$$
S(r, f)=o(T(r, f)),
$$

except possibly for a finite length of $r$-values. In particular, if $f(z)$ is of finite order, the error term $S(r, f)$ grows at most like $O(\log r)$ without an exceptional set. For infinite order functions, however, in general the exceptional set in (110) cannot be deleted [55].

In loose terms, Nevanlinna's second main theorem implies that the counting function $N(r, a)$ must usually be much larger than the proximity function $m(r, a)$ in the sum (44). In order to measure the deviation from regular value distribution more precisely, Nevanlinna introduced the deficiency

$$
\delta(a, f)=1-\limsup _{r \rightarrow \infty} \frac{N(r, a)}{T(r, f)}
$$

and the ramification index

$$
\theta(a, f)=\liminf _{r \rightarrow \infty} \frac{N(r, a)-\bar{N}(r, a)}{T(r, f)},
$$

where $\bar{N}(r, a)$ is the integrated counting function for the $a$-points of $f(z)$, ignoring multiplicities. The point $a \in \mathbb{C}$ is called a deficient value of $f(z)$ if $\delta(a, f)>0$, and a ramified value of $f(z)$ if $\theta(a, f)>0$. Moreover, it is said that $a$ is completely ramified if all $a$-points of $f(z)$ have multiplicity 2 or higher.

Clearly, the deficiency satisfies $0 \leqslant \delta(a, f) \leqslant 1$ for all $a \in \mathbb{C} \cup\{\infty\}$. Moreover, by the second main theorem (110) it follows that for any meromorphic function $f(z)$ there can be at most countably many deficient values [54], and

$$
\sum_{a \in \mathbb{C} \cup\{\infty\}}(\delta(a, f)+\theta(a, f)) \leqslant 2 .
$$

Inequality (112) is a deep generalization of Picard's theorem, which, translated to the language of Nevanlinna theory, says only that the counting function $N(r, a)$ may be identically zero for at most two values $a$ in the extended complex plane $\mathbb{C} \cup\{\infty\}$. Borel's theorem states, on the other hand, that for any entire finite-order function $f(z)$ the deficiency $\delta(a, f)$ may be identically zero for at most two values $a$. Therefore the second main theorem is not only a generalization of Borel's result to meromorphic functions, but it tells much more on the value distribution of entire functions as well.

A natural problem connected to relation (112) is to find a meromorphic function $f(z)$ which at prescribed points has certain non-zero deficiencies $\delta(a, f)$ and ramification indices $\theta(a, f)$. This is the inverse problem for the deficiency relation, originally proposed and partially solved by Nevanlinna himself (see, for instance, [93]). The problem was finally completely solved by Drasin [26] by the means of quasi-conformal mappings.

\subsection{Second main theorem and Picard's theorem for the shift operator}

In this subsection we present a difference analogue of the second main theorem of Nevanlinna theory and some of its applications, including a Picard-type theorem for the shift operator. For proofs of the theorems and further discussion, see [47]. 
The following theorem is an analogue of the second main theorem (110), where the ramification term $N_{1}(r, f)$ has been replaced by a quantity depending on exact differences of a finite-order meromorphic function [47].

Theorem 7.3. Let $c \in \mathbb{C}$, and let $f(z)$ be a meromorphic function of finite order such that $\Delta_{c} f \not \equiv 0$. Let $q \geqslant 2$, and let $a_{1}, \ldots, a_{q}$ be distinct complex constants. Then

$$
m(r, f)+\sum_{k=1}^{q} m\left(r, \frac{1}{f-a_{k}}\right) \leqslant 2 T(r, f)-N_{\text {pair }}(r, f)+S(r, f),
$$

where

$$
N_{\text {pair }}(r, f):=2 N(r, f)-N\left(r, \Delta_{c} f\right)+N\left(r, \frac{1}{\Delta_{c} f}\right)
$$

We will now discuss some of the implications of theorem 7.3. To this end, we assume that from now on $f(z)$ is a meromorphic function of finite order such that $f(z+c) \not \equiv f(z)$. We define $n_{c}(r, a), a \in \mathbb{C}$, to be the number of points $z_{0}$ in the disc of radius $r$ centred at the origin such that $f\left(z_{0}\right)=a$ and $f\left(z_{0}+c\right)=a$, where each point is counted according to the number of equal terms in the beginning of Taylor series expansions of $f(z)$ and $f(z+c)$ in a neighbourhood of $z_{0}$. We call such points c-separated a-pairs of $f(z)$ in the $\operatorname{disc}\{z:|z| \leqslant r\}$. For instance, if in a neighbourhood of $z_{0}$,

$$
f(z)=a+c_{1}\left(z-z_{0}\right)+\alpha\left(z-z_{0}\right)^{2}+O\left(\left(z-z_{0}\right)^{3}\right)
$$

and

$$
f(z+c)=a+c_{1}\left(z-z_{0}\right)+\beta\left(z-z_{0}\right)^{2}+O\left(\left(z-z_{0}\right)^{3}\right)
$$

where $\alpha \neq \beta$, then the point $z_{0}$ is counted two times in $n_{c}(r, a)$. The number of $c$-separated pole pairs, $n_{c}(r, \infty)$, of $f(z)$ is the same as the number of $c$-separated 0 -pairs of $1 / f(z)$. This means that if $f(z)$ has a pole with multiplicity $p$ at $z_{0}$ and another pole with multiplicity $q$ at $z_{0}+c$ then this pair is counted $\min \{p, q\}+m$ times in $n_{c}(r, \infty)$, where $m$ is the number of equal terms in the beginning of the Laurent series expansions of $f(z)$ and $f(z+c)$ in a neighbourhood of $z_{0}$.

Note that since $f(z)$ was assumed not to be a periodic function with period $c$, it follows that $n_{c}(r, a)$ is finite for any finite $r$. Otherwise there would be a point $z_{0} \in \mathbb{C}$ in a neighbourhood of which the series expansions of $f(z)$ and $f(z+c)$ would be identical. But this means that $f(z) \equiv f(z+c)$ in the whole complex plane, which contradicts the non-periodicity of $f(z)$. However, it is possible that $n_{c}(r, a)$ is strictly greater than the counting function $n(r, a)$.

We now define the integrated counting function for the $c$-separated $a$-pairs as

$$
N_{c}(r, a):=\int_{0}^{r} \frac{n_{c}(t, a)-n_{c}(0, a)}{t} \mathrm{~d} t+n_{c}(0, a) \log r
$$

where $a \in \mathbb{C} \cup\{\infty\}$. A natural difference analogue of $\bar{N}(r, a)$ is then

$$
\tilde{N}_{c}(r, a):=N(r, a)-N_{c}(r, a)
$$

which, roughly speaking, counts the number of those $a$-points of $f(z)$ which are not $c$-separated pairs. It is immediately clear by definition that the inequalities $0 \leqslant \bar{N}(r, a) \leqslant T(r, f)$ and $\widetilde{N}_{c}(r, a) \leqslant T(r, f)$ are valid for any meromorphic function $f(z)$ and any $a \in \mathbb{C} \cup\{\infty\}$, as long as $r$ is sufficiently large. However, the counting function $\widetilde{N}_{c}(r, a)$ may, for some values $a$, be negative for all $r$ large enough. For instance, consider the function $g(z):=\wp(z)+\exp (z)$ where $\wp(z)$ is a Weierstrass elliptic function with a period $c \neq 2 \pi \mathrm{i}$. Then $T(r, g)=N(r, g)+S(r, g)$ and each pole of $g(z)$ contributes 2 to $n(r, g)$ but -2 to 
$\tilde{n}_{c}(r, g)$. Therefore $\tilde{N}_{c}(r, g)=-T(r, g)+S(r, g)$. The following theorem yields a lower bound for the growth of the counting function $\widetilde{N}_{c}(r, a)$ and implies that $\widetilde{N}_{c}(r, a)$ must be relatively large for most values $a \in \mathbb{C} \cup\{\infty\}$.

Theorem 7.4. Let $c \in \mathbb{C}$, and let $f(z)$ be a meromorphic function of finite order such that $\Delta_{c} f \not \equiv 0$. Let $q \geqslant 2$, and let $a_{1}, \ldots, a_{q}$ be distinct complex constants. Then

$$
(q-1) T(r, f) \leqslant \widetilde{N}_{c}(r, f)+\sum_{k=1}^{q} \widetilde{N}_{c}\left(r, \frac{1}{f-a_{k}}\right)+S(r, f) .
$$

Theorem 7.4 implies that $\tilde{N}_{c}(r, a) \geqslant-T(r, f)+S(r, f)$ for all $a \in \mathbb{C} \cup\{\infty\}$, and $\tilde{N}_{c}(r, a)=T(r, f)+S(r, f)$ for all except at most countably many values $a \in \mathbb{C} \cup\{\infty\}$ (see, for instance, [54] for a method of proving the latter relation). This means, by the definition of $\widetilde{N}_{c}(r, a)$, that points appearing pairwise, or in infinite lines, separated from each other by the constant vector $c \in \mathbb{C}$, are in a sense exceptional. For instance, by theorem 7.4 any finite-order meromorphic function $f(z)$ is either periodic with period $c$, or it can have at most one non-deficient value $a$ such that whenever $f(z)=a$ also $f(z+c)=a$ and the first two terms in the series expansions of $f(z)$ at $z$ and $z+c$ are identical (such as the function $g(z)=\wp(z)+\exp (z)$ discussed above).

In order to analyse this phenomenon more precisely, we introduce some additional notation. A difference analogue of the index of multiplicity $\theta(a, f)$ is called the $c$-separated pair index, and it is defined by

$$
\pi_{c}(a, f):=\liminf _{r \rightarrow \infty} \frac{N_{c}(r, a)}{T(r, f)}
$$

where $a \in \mathbb{C} \cup\{\infty\}$. Similarly, we define

$$
\Pi_{c}(a, f):=1-\limsup _{r \rightarrow \infty} \frac{\widetilde{N}_{c}(r, a)}{T(r, f)},
$$

which is an analogue of

$$
\Theta(a, f)=1-\limsup _{r \rightarrow \infty} \frac{\bar{N}(r, a)}{T(r, f)}
$$

in the usual value distribution theory. With this notation in hand, theorem 7.4 yields the following analogue of the deficiency relation (112).

Corollary 7.5. Let $c \in \mathbb{C}$, and let $f(z)$ be a meromorphic function of finite order such that $\Delta_{c} f \not \equiv 0$. Then $\Pi_{c}(a, f)=0$ except for at most countably many $a \in \mathbb{C} \cup\{\infty\}$, and

$$
\sum_{a}\left(\delta(a, f)+\pi_{c}(a, f)\right) \leqslant \sum_{a} \Pi_{c}(a, f) \leqslant 2 .
$$

In the classical Nevanlinna theory it is clear by (114) that $0 \leqslant \Theta(a, f) \leqslant 1$ for all meromorphic functions $f(z)$ and for all $a$ in the extended complex plane. For the difference analogue $\Pi_{c}(a, f)$, however, it is not immediately clear that a uniform upper bound even exists. The fact that $\Pi_{c}(a, f) \leqslant 2$ for all $a$ follows by corollary 7.5 , and the maximal deficiency sum

$$
\sum_{a} \Pi_{c}(a, f)=2
$$


may as a matter of fact be attained by a single value $a$. For instance, the function $g(z)=\wp(z)+\exp (z)$, where $\wp(z)$ is a Weierstrass elliptic function with a period $c \neq 2 \pi \mathrm{i}$, satisfies $\Pi_{c}(\infty, g)=2$.

We say that $a$ is an exceptional paired value of $f(z)$ with the separation $c$ if the following property holds for all except at most finitely many a-points of $f(z)$ : whenever $f(z)=a$ then also $f(z+c)=a$ with the same or higher multiplicity. Now, in a similar way as the second main theorem of Nevanlinna theory implies Picard's theorem, theorem 7.4 yields a shift analogue of Picard's theorem.

Corollary 7.6. If a finite-order meromorphic function $f(z)$ has three exceptional paired values with the separation $c$, then $f(z)$ is a periodic function with period $c$.

Since by definition all Picard exceptional values of $f(z)$ are also exceptional paired values, corollary 7.6 in fact yields the classical Picard's theorem for finite-order meromorphic functions. Corollary 7.6 also implies that if a finite-order meromorphic function $f(z)$ has two non-empty groups of three exceptional paired values with two different separations independent over the reals, say $c_{1}$ and $c_{2}$, then either $f(z)$ is a constant or $f(z)$ is an elliptic function with periods $c_{1}$ and $c_{2}$.

Concerning the sharpness of corollary 7.6, consider the elliptic function $\operatorname{sn}(z, k)$, where $k \in(0,1)$ is the elliptic modulus and $K$ is the complete elliptic integral. The function $\operatorname{sn}(z, k)$ is periodic with the periods $4 K$ and $2 \mathrm{i} K^{\prime}$, and it attains the value zero at points $2 n K+2 m \mathrm{i} K^{\prime}$ and has its poles at $2 n K+(2 m+1) \mathrm{i} K^{\prime}$, where $n, m \in \mathbb{Z}$. Therefore $\operatorname{sn}(z, k)$ has exactly two exceptional paired values with the separation $2 K$, which is the maximal amount by corollary 7.6. Also, the function $f(z)=\operatorname{sn}(z, k)$ is another example of a finite-order meromorphic function satisfying (112).

The restriction to finite-order meromorphic functions in corollary 7.6 (or corollary 7.5) cannot be removed, since $\exp (\exp (z))$ has three exceptional paired values with the separation $\log 2$. In addition to the Picard exceptional zeros and poles, the value 1 is exceptionally paired.

Analogously to complete ramification, we say that a point $a$ is completely paired with the separation $c$ if whenever $f(z)=a$ then either $f(z+c)=a$ or $f(z-c)=a$, with the same multiplicity.

Corollary 7.7. Let $c \in \mathbb{C}$, and let $f(z)$ be a meromorphic function of finite order such that $\Delta_{c} f \not \equiv 0$. Then $f(z)$ has at most four completely paired points with separation $c$.

As in corollary 7.7, a non-periodic finite-order function $f(z)$ can have at most three values $a$ which only appear in lines of three (i.e. such that for some $z_{0} \in \mathbb{C}, f\left(z_{0}\right) \neq a, f\left(z_{0}+j c\right)=a$ with the same multiplicity for each $j=1,2,3$, and $f\left(z_{0}+4 c\right) \neq a$ ), and a maximum of two values which appear only in lines of four or more.

\subsection{Meromorphic functions sharing values}

Another consequence of Nevanlinna's second main theorem is the five value theorem, which says that if two non-constant meromorphic functions $f(z)$ and $g(z)$ share five values ignoring multiplicity then these functions must be identical. Nevanlinna also showed that if $f(z)$ and $g(z)$ are distinct, they can share four values counting multiplicities only in some very special cases which can be listed explicitly. For further studies concerning functions sharing three or four values see, for instance [42, 43, 91, 123].

By ignoring paired points instead of multiplicity, we obtain a difference analogue of the five value theorem. To be exact, we say that two meromorphic functions $f(z)$ and $g(z)$ 
share a point $a$, ignoring $c$-separated pairs, when $f(z)=a$ if and only if $g(z)=a$ with the same multiplicity, unless $a$ is a $c$-separated pair of $f(z)$ or $g(z)$. In short, all paired points are ignored when determining whether or not $f(z)$ and $g(z)$ share $a$. This also means that if $f(z)$ has a paired $a$-point at $z_{0}$ and $g(z)$ has a single $a$-point at the same location, this point is not shared by $f(z)$ and $g(z)$.

Theorem 7.8. Let $c \in \mathbb{C}$, and let $f(z)$ and $g(z)$ be meromorphic functions of finite order. If there are five distinct points $a_{k}$ such that $f(z)$ and $g(z)$ share $a_{k}$, ignoring c-separated pairs, for all $k=1, \ldots, 5$ then either $f(z) \equiv g(z)$ or both $f(z)$ and $g(z)$ are periodic with period c.

The elliptic functions $\operatorname{sn} z$ and $1 /$ sn $z$ share the points 1 and -1 , counting multiplicities, and for both functions zero and infinity are exceptional paired values with separation $2 K$. Therefore, $\operatorname{sn} z$ and $1 / \operatorname{sn} z$ share the points $-1,0,1$ and $\infty$ ignoring pairs, which shows that the number five cannot be replaced by four in theorem 7.8.

\section{8. $q$-difference equations}

By a $q$-difference equation we mean an equation of the form $P(z, f)=0$, where $P(z, f)$ is polynomial in $f$ and finitely many of its $q$-shifts $f\left(q^{n} z\right), n \in \mathbb{Z}$. The study of meromorphic solutions of $q$-difference equations has been ongoing since the 19th century. Valiron has shown that the non-autonomous Schröder $q$-difference equation

$$
f(q z)=R(z, f(z))
$$

where $R(z, f(z))$ is rational in both arguments and nonlinear in $f(z)$, has a one parameter family of meromorphic solutions, if $q \in \mathbb{C}$ is suitably chosen [125]. Gundersen et al [45] showed that all meromorphic solutions of (117) are of order $\log _{q}\left(\operatorname{deg}_{f} R\right)$, where $\log _{q}$ denotes the $q$-based logarithm. Their result implies a $q$-difference analogue of a classical result due to Malmquist [85]. If the $q$-difference equation (117) admits a meromorphic solution of order zero, then (117) reduces to a $q$-difference Riccati equation, i.e. $\operatorname{deg}_{f} R=1$.

There are a number of effective methods from different areas of complex analysis which can be applied to study the value distribution of meromorphic solutions of the Schröder equation (117), and $q$-difference equations in general. For instance, Eremenko and Sodin [28] used methods from complex dynamics to show that the Valiron and Nevanlinna deficient values of meromorphic solutions of the autonomous Schröder equation (117) always coincide with the exceptional values of $R(z)$. On the other hand, Ishizaki and Yanagihara [66] showed that this is not true in general for the non-autonomous Schröder equation. They have also studied Borel and Julia directions of meromorphic solutions of the Schröder equation by using methods from Nevanlinna theory [68, 69].

Just as equation (117) admits meromorphic solutions only for a special choice of $q$, the coefficients of a linear $q$-difference equation must satisfy certain conditions to guarantee the existence of meromorphic solutions. Bergweiler, Ishizaki and Yanagihara found sufficient conditions for the existence, and characterized the growth of meromorphic solutions of linear $q$-difference equations in terms of the Nevanlinna characteristic [9]. They concluded that all meromorphic solutions $f(z)$ of a linear $q$-difference equation with rational coefficients satisfy $T(r, f)=O\left((\log r)^{2}\right)$ and $(\log r)^{2}=O(T(r, f))$. For entire solutions there are other methods, for instance, a $q$-shift analogue of the Wiman-Valiron theory by Bergweiler, Ishizaki and Yanagihara [10], and a functional analytic method by Ramis [112], by which the asymptotic behaviour of solutions may be characterized even more precisely.

The zero distribution of entire solutions of linear $q$-difference equations has been studied by Bergweiler and Hayman [8]. They showed that, under a certain condition, solutions behave 
asymptotically like products of $\theta$-functions, which enabled them to deduce an asymptotic formula for the locations of zeros. Heittokangas et al [59] studied the distribution of zeros and poles of meromorphic solutions of linear $q$-difference equations by using methods from Nevanlinna theory. Although their method does not give the locations of the poles and zeros explicitly, it can be used to obtain information on the relative densities of zeros and poles of solutions compared to the growth of the solution.

In section 5.2 we gave a number of results on Nevanlinna theory for finite-order meromorphic functions related to the shift operator $\Delta_{c} f=f(z+c)-f(z)$. Similar results may be obtained for the $q$-difference operator $\Delta_{q} f=f(q z)-f(z)$, provided that $f(z)$ is a meromorphic function of zero order [4]. The restriction to zero-order meromorphic functions is analogous to demanding finite order of growth in the ordinary shift case. For instance, all meromorphic solutions of linear and $q$-Riccati difference equation are of zero order. The following theorem is a $q$-shift analogue of theorem 5.6.

Theorem 8.1. Let $f(z)$ be a non-constant zero-order meromorphic function, and $q \in \mathbb{C} \backslash\{0\}$. Then

$$
m\left(r, \frac{f(q z)}{f(z)}\right)=o(T(r, f))
$$

for all $r$ on a set $E$ of logarithmic density 1, i.e. on a set E such that

$$
\limsup _{r \rightarrow \infty} \frac{\int_{E \cap[0, r)} \frac{\mathrm{d} t}{t}}{\log r}=1 .
$$

Theorem 8.1 may be used to obtain a set of tools for analysing zero-order meromorphic solutions of $q$-difference equations in a similar fashion as theorem 5.6 in the case of difference equations. The procedure is similar to that already described in section 7 [4].

\section{Future work and conclusions}

Nevanlinna theory is only one of several approaches to the discrete/difference Painlevé equations. Being complex analytic in nature, it is very much in the spirit of the original Painlevé property for differential equations.

There are many important open problems in this area. We list some below.

(i) Prove the existence of finite-order meromorphic solutions for difference Painlevé equations and characterize the remaining freedom in the periodic functions.

(ii) Determine which classes of rational difference equations admit meromorphic solutions and characterize them (e.g., how many are there?)

(iii) Does the existence of sufficiently many finite-order meromorphic solutions guarantee the existence of a Lax pair?

(iv) Classify equations whose only finite-order meromorphic solutions are non-admissible.

(v) Develop connections with other approaches (algebraic entropy, Diophantine integrability, affine Weyl algebras and Bäcklund transformations).

(vi) Study the existence of finite-order meromorphic solutions of higher dimensional (i.e. lattice) equations-e.g., dKdV, dKP, etc.

(vii) Determine in what sense the solutions of the difference Painlevé equations define new transcendents. 


\section{Acknowledgments}

The first author was supported by an EPSRC Advanced Research Fellowship and a grant number F/00 261/N from the Leverhulme Trust. The second author was partially supported by the Academy of Finland grants 105795, 118314 and 210245.

\section{References}

[1] Ablowitz M J, Halburd R G and Herbst B 2000 On the extension of the Painlevé property to difference equations Nonlinearity 13 889-905

[2] Azarina Yu V 1990 Meromorphic solutions of the equation $w(z+1)=R(w(z)) J$. Sov. Math. $49871-5$ (Original Russian version: Teor. Funktsi Funktsional. Anal. i. Prilozhen 48 (1987) 26-32)

[3] Baker I N and Liverpool L S O 1984 The entire solutions of a polynomial difference equation Aequationes Math. 27 97-113

[4] Barnett D, Halburd R G, Korhonen R and Morgan W Applications of Nevanlinna theory to q-difference equations Proc. R. Soc. Edinburgh A at press

[5] Batchelder P M 1967 An Introduction to Linear Difference Equations (New York: Dover)

[6] Baxter R J 1982 Exactly Solved Models in Statistical Mechanics (London: Academic)

[7] Bellon M P and Viallet C-M 1999 Algebraic entropy Commun. Math. Phys. 204 425-37

[8] Bergweiler W and Hayman W 2003 Zeros of solutions of a functional equation Comput. Methods Funct. Theory 3 55-78

[9] Bergweiler W, Ishizaki K and Yanagihara N 1998 Meromorphic solutions of some functional equations Methods Appl. Anal. 5 248-58 (Correction: Methods Appl. Anal. 6 1999)

[10] Bergweiler W, Ishizaki K and Yanagihara N 2002 Growth of meromorphic solutions of some functional equations. I Aequationes Math. 63 140-51

[11] Bergweiler W and Langley J K Zeros of differences of meromorphic functions Math. Proc. Cambridge Philos. Soc. at press (Preprint math.CV/0506441)

[12] Bessis D, Itzykson C and Zuber J-B 1980 Quantum field theory techniques in graphical enumeration $A d v$. Appl. Math. 1 109-57

[13] Birkhoff G D 1911 General theory of linear difference equations Trans. Am. Math. Soc. 12 243-84

[14] Birkhoff G D 1913 The generalized Riemann problem for linear differential equations and the allied problems for linear difference and $q$-difference equations Proc. Am. Acad. Arts Sci. 49 521-68

[15] Blumenthal O 1910 Principes de la théorie des fonctions entières d'ordre infini Collection de Monographies sur la théorie des Fonctions (Paris: Gauthier-Villars)

[16] Borel É 1897 Sur les zéros des fonctions entières Acta Math. 20 357-96

[17] Brézin E and Kazakov V A 1990 Exactly solvable field theories of closed strings Phys. Lett. B 236 144-50

[18] Carne T K 1986 Brownian motion and Nevanlinna theory Proc. Lond. Math. Soc. 52 349-68

[19] Cherry W and Ye Z 2001 Nevanlinna's Theory of Value Distribution (Berlin: Springer)

[20] Chiang Y M and Feng S J 2006 On the Nevanlinna characteristic of $f(z+\eta)$ and difference equations in the complex plane Ramanujan J. at press (Preprint math.CV/0609324)

[21] Chiang Y M and Ruijsenaars S N M 2006 On the Nevanlinna order of meromorphic solutions to linear analytic difference equations Stud. Appl. Math. 116 (3) 257-87

[22] Chuang C T 1993 Normal Families of Meromorphic Functions (River Edge, NJ: World Scientific)

[23] Clunie J 1962 On integral and meromorphic functions J. Lond. Math. Soc. 37 17-27

[24] Costin O and Kruskal M 2002 Movable singularities of solutions of difference equations in relation to solvability and a study of a superstable fixed point Theor. Math. Phys. 133 1455-62

[25] Douglas M R and Shenker S H 1990 Strings in less than one dimension Nucl. Phys. B 335 635-54

[26] Drasin D 1977 The inverse problem of the Nevanlinna theory Acta Math. 138 83-151

[27] Eremenko A E 1982 Meromorphic solutions of algebraic differential equations Uspekhi Mat. Nauk 37 53-82, 240 (Translation in Russ. Math. Surv.)

[28] Eremenko A E and Sodin M L 1990 Iterations of rational functions and the distribution of the values of Poincaré functions Teor. Funktsii Funktsional. Anal. i Prilozhen. 53 18-25 (Russian) (Translation in J. Sov. Math. 58 (1992) 504-9)

[29] Falqui G and Viallet C-M 1993 Singularity, complexity, and quasi-integrability of rational mappings Commun. Math. Phys. 154 111-25

[30] Fatou P 1920 Sur les equations fonctionnelles Bull. Soc. France 48 33-94, 208-314

[31] Fokas A S, Grammaticos B and Ramani A 1993 From continuous to discrete Painlevé equations J. Math. Anal. Appl. 180 342-60 
[32] Fokas A S, Its A R and Kitaev A V 1991 Discrete Painlevé equations and their appearance in quantum gravity Commun. Math. Phys. 142 313-44

[33] Freud G 1976 On the coefficients in the recursion formulae of orthogonal polynomials Proc. R. Ir. Acad. A 76 $1-6$

[34] Goldberg A A, Levin B Ya and Ostrovskii I V 1997 Entire and meromorphic functions Complex Analysis I. Encyclopaedia Math. Sci. vol 85 (Berlin: Springer) pp 1-193, 254-61

[35] Grammaticos B, Nijhoff F W and Ramani A 1999 Discrete Painlevé equations The Painlevé Property (CRM Ser. Math. Phys.) ed R Conte (New York: Springer) pp 413-516

[36] Grammaticos B, Ohta Y, Ramani A and Sakai H 1998 Degeneration through coalescence of the $q$-Painlevé VI equation J. Phys. A: Math. Gen. 31 3545-58

[37] Grammaticos B and Ramani A 1993 Discrete Painlevé equations: derivation and properties Applications of Analytic and Geometric Methods to Nonlinear Differential Equations (NATO ASI Series C: Mathematical and Physical Sciences vol 413) ed P A Clarkson (Dordrecht: Kluwer) pp 299-313

[38] Grammaticos B, Ramani A and Papageorgiou V 1991 Do integrable mappings have the Painlevé property? Phys. Rev. Lett. 67 1825-8

[39] Grammaticos B, Ramani A and Papageorgiou V 1997 Discrete dressing transformations and Painlevé equations Phys. Lett. A 235 475-9

[40] Gromak V I, Laine I and Shimomura S 2002 Painlevé Differential Equations in the Complex Plane (Berlin: Walter de Gruyter)

[41] Gross D and Migdal A 1990 Nonperturbative two-dimensional quantum gravity Phys. Rev. Lett. 64 127-30

[42] Gundersen G G 1979 Meromorphic functions that share three or four values J. Lond. Math. Soc. 20 457-66

[43] Gundersen G G 1983 Meromorphic functions that share four values Trans. Am. Math. Soc. 277 545-67 (Correction: Trans. Am. Math. Soc. 304 (1987) 847-50)

[44] Gundersen G G 1988 Estimates for the logarithmic derivative of meromorphic functions, plus similar estimates J. Lond. Math. Soc. 37 88-104

[45] Gundersen G G, Heittokangas J, Laine I, Rieppo J and Yang D 2002 Meromorphic solutions of generalized Schröder equations Aequationes Math. 63 110-35

[46] Halburd R G 2005 Diophantine integrability J. Phys. A: Math. Gen. 38 L263-9

[47] Halburd R G and Korhonen R J 2005 Nevanlinna theory for the difference operator Ann. Acad. Sci. Fenn. Math. 31 463-78

[48] Halburd R G and Korhonen R J 2006 Difference analogue of the lemma on the logarithmic derivative with applications to difference equations J. Math. Anal. Appl. 314 477-87

[49] Halburd R G and Korhonen R J 2006 Existence of finite order meromorphic solutions as a detector of integrability in difference equations Physica D 218 191-203

[50] Halburd R G and Korhonen R J 2005 Finite-order meromorphic solutions and the discrete Painlevé equations Proc. Lond. Math. Soc. at press (Preprint nlin.SI/0504026)

[51] Harris W A and Sibuya Y 1964 Asymptotic solutions of systems of nonlinear difference equations Arch. Ration. Mech. Anal. 15 377-95

[52] Harris W A and Sibuya Y 1965 General solution of nonlinear difference equations Trans. Am. Math. Soc. 115 62-75

[53] Harris W A and Sibuya Y 1966 On asymptotic solutions of systems of nonlinear difference equations J. Reine Angew. Math. 222 120-35

[54] Hayman W K 1964 Meromorphic Functions (Oxford: Clarendon Press)

[55] Hayman W K 1965 On the characteristic of functions meromorphic in the plane and of their integrals Proc. Lond. Math. Soc. 14a 93-128

[56] Hayman W K 1974 The local growth of power series: a survey of the Wiman-Valiron method Canad. Math. Bull. 17 317-58

[57] Hayman W K 1982 Rolf Nevanlinna Bull. Lond. Math. Soc. 14 419-36

[58] Heittokangas J, Korhonen R, Laine I, Rieppo J and Tohge K 2001 Complex difference equations of Malmquist type Comput. Methods Funct. Theory $127-39$

[59] Heittokangas J, Laine I, Rieppo J and Yang D 2000 Meromorphic solutions of some linear functional equations Aequationes Math. 60 148-66

[60] Henson C W and Rubel L A 1984 Some applications of Nevanlinna theory to mathematical logic: identities of exponential functions Trans. Am. Math. Soc. 282 1-32

[61] Hietarinta J and Viallet C-M 1998 Singularity confinement and chaos in discrete systems Phys. Rev. Lett. $81325-8$

[62] Hille E 1976 Ordinary Differential Equations in the Complex Domain (New York: Wiley-Interscience)

[63] Hirai I 1979 On a meromorphic solution of some difference equation J. Coll. Arts Sci., Chiba Univ. 12 5-10 
[64] Iatrou A and Roberts J A G 2001 Integrable mappings of the plane preserving biquadratic invariant curves J. Phys. A: Math. Gen. 34 6617-36

[65] Immink G K 1984 Asymptotics of Analytic Difference Equations (Lecture Notes in Mathematics vol 1085) (Berlin: Springer)

[66] Ishizaki K and Yanagihara N 2004 Deficiency for meromorphic solutions of Schröder equations Complex Var. Theory Appl. 49 539-48

[67] Ishizaki K and Yanagihara N 2004 Wiman-Valiron method for difference equations Nagoya Math. J. 175 75-102

[68] Ishizaki K and Yanagihara N 2005 Borel and Julia directions of meromorphic Schröder functions Math. Proc. Camb. Phil. Soc. 139 139-47

[69] Ishizaki K and Yanagihara N 2005 Remarks on deficiencies for meromorphic Schröder functions Proc. 12th International Conference on Finite or Infinite Dimensional Complex Analysis and Applications (Kyushu: Kyushu University Press) pp 105-12

[70] Its A R, Kitaev A V and Fokas A S 1990 The isomonodromy approach in the theory of two-dimensional quantum gravitation Russ. Math. Surv. 45 155-7

[71] Itzykson C and Zuber J B 1980 The planar approximation: II. J. Math. Phys. 21 411-21

[72] Jank G and Volkmann L 1985 Einführung in die Theorie der ganzen und meromorphen Funktionen mit Anwendungen auf Differentialgleichungen (Basel: Birkhäuser)

[73] Jimbo M, Sakai H, Ramani A and Grammaticos B 1996 Bilinear structure and Schlesinger transforms of the $q-\mathrm{p}_{I I I}$ and $q-\mathrm{p}_{I V}$ equations Phys. Lett. A $217111-8$

[74] Joshi N, Burtonclay D and Halburd R 1992 Nonlinear nonautonomous discrete dynamical systems from a general discrete isomonodromy problem Lett. Math. Phys. 26 123-31

[75] Julia G 1918 Memoire sur l'iteration des fonctions rationnelles J. Math. Pures Appl. 1 47-245

[76] Kajiwara K, Ohta Y and Satsuma J 1995 Casorati determinant solutions for the discrete Painlevé-III equation J. Math. Phys. 36 4162-74

[77] Kajiwara K, Ohta Y, Satsuma J, Grammaticos B and Ramani A 1994 Casorati determinant solutions for the discrete Painlevé-II equation J. Phys. A: Math. Gen. 27 915-22

[78] Kimura T 1971 On the iteration of analytic functions Funkcial. Ekvac. 14 197-238

[79] Laine I 1993 Nevanlinna Theory and Complex Differential Equations (Berlin: Walter de Gruyter)

[80] Laine I, Rieppo J and Silvennoinen H 2005 Remarks on complex difference equations Comput. Methods Funct. Theory 5 77-88

[81] Lehto O 1982 On the birth of the Nevanlinna theory Ann. Acad. Sci. Fenn. Ser. A I Math. 7 5-23

[82] Levy H and Lessman F 1992 Finite Difference Equations (New York: Dover)

[83] Magnus A P 1995 Painlevé type differential equations for the recurrence coefficients of semiclassical orthogonal polynomials J. Comput. Appl. Math. 57 215-37

[84] Magnus A P 1999 Freud's equations for orthogonal polynomials as discrete Painlevé equations Symmetries and Integrability of Difference Equations (Canterbury, 1996) (London Math. Soc. Lecture Notes Series) (Cambridge: Cambridge University Press) pp 228-43

[85] Malmquist J 1913 Sur les fonctions á un nombre fini des branches définies par les équations différentielles du premier ordre Acta Math. 36 297-343

[86] McMillan E M 1971 A problem in the stability of periodic systems Topics in Modern Physics, a Tribute to E.V. Condon ed E Brittin and H Odabasi (Boulder, CO: Colorado Assoc. Univ. Press) pp 219-44

[87] Meschkowski H 1959 Differenzengleichungen (Göttingen: Vandenhoeck \& Ruprecht)

[88] Milne-Thomson L M 1951 The Calculus of Finite Differences (London: Macmillan)

[89] Mohon'ko A Z 1971 The Nevanlinna characteristics of certain meromorphic functions Teor. Funktsii Funktsional. Anal. i Prilozhen 14 83-7 (Russian)

[90] Mohon'ko A Z and Mohon'ko V D 1974 Estimates of the Nevanlinna characteristics of certain classes of meromorphic functions, and their applications to differential equations Sibirsk. Mat. Zh. 15 1305-22 (Russian)

[91] Mues E 1989 Meromorphic functions sharing four values Complex Var. Theory Appl. 12 169-79

[92] Nevanlinna R 1925 Zur Theorie der meromorphen Funktionen Acta Math. 46 1-99

[93] Nevanlinna R 1974 Eindeutige analytische Funktionen (Die Grundlehren der mathematischen Wissenschaften vol 46) (Berlin: Springer)

[94] Nijhoff F W and Papageorgiou V G 1991 Similarity reductions of integrable lattices and discrete analogues of the Painlevé II equation Phys. Lett. A $153337-44$

[95] Nörlund N E 1924 Vorlesungen über Differenzenrechnung (Die Grundlehren der mathematischen Wissenschaften vol 13) (Berlin: Springer)

[96] Ohta Y, Tamizhmani K M, Grammaticos B and Ramani A 1999 Singularity confinement and algebraic entropy: the case of the discrete Painlevé equations Phys. Lett. A 262 152-7 
[97] Osgood C F 1981 A number theoretic-differential equations approach to generalizing Nevanlinna theory Indian J. Math. 23 1-15

[98] Osgood C F 1984 Effective Bounds on the Diophantine Approximation of Algebraic Functions, and Nevanlinna Theory (Lecture Notes in Mathematics vol 1052) (Berlin: Springer)

[99] Osgood C F 1985 Sometimes effective Thue-Siegel-Roth-Schmidt-Nevanlinna bounds, or better J. Number Theory 21 347-89

[100] Papageorgiou V G, Nijhoff F W, Grammaticos B and Ramani A 1992 Isomonodromic deformation problems for discrete analogues of Painlevé equations Phys. Lett. A 164 57-64

[101] Periwal V and Shevitz D 1990 Unitary matrix models as exactly solvable string theories Phys. Rev. Lett. 64 1326-9

[102] Picard É 1880 Mémoire sur les fonctions entières Ann. École. Norm. 9 145-66

[103] Praagman C 1986 Fundamental solutions for meromorphic linear difference equations in the complex plane, and related problems J. Reine Angew. Math. 369 101-9

[104] Quispel G R W 2004 An alternating integrable map whose square is the QRT map Phys. Lett. A 307 50-4

[105] Quispel G R W, Roberts J A G and Thompson C J 1988 Integrable mappings and soliton equations Phys. Lett. A $126419-21$

[106] Quispel G R W, Roberts J A G and Thompson C J 1989 Integrable mappings and soliton equations II Physica D 34 183-92

[107] Ramani A, Carstea A S, Grammaticos B and Ohta Y 2002 On the autonomous limit of discrete Painlevé equations Physica A 305 437-44

[108] Ramani A and Grammaticos B 1996 Discrete Painlevé equations: coalescences, limits and degeneracies Physica A 228 160-71

[109] Ramani A, Grammaticos B and Hietarinta J 1991 Discrete versions of the Painlevé equations Phys. Rev. Lett. 67 1829-32

[110] Ramani A, Grammaticos B and Karra G 1992 Linearizable mappings Physica A 181 115-27

[111] Ramani A, Grammaticos B, Tamizhmani T and Tamizhmani K M 2003 The road to the discrete analogue of the Painlevé property: Nevanlinna meets singularity confinement Comput. Math. Appl. 45 1001-12

[112] Ramis J-P 1992 About the growth of entire functions solutions of linear algebraic $q$-difference equations Ann. Fac. Sci. Toulouse Math. 153-94

[113] Roberts J A G and Vivaldi F 2003 Arithmetical method to detect integrability in maps Phys. Rev. Lett. 90034102

[114] Röhrl H 1962 Holomorphic fiber bundles over Riemann surfaces Bull. Am. Math. Soc. 68 125-60

[115] Ru M 2001 Nevanlinna Theory and its Relation to Diophantine Approximation (River Edge, NJ: World Scientific)

[116] Ruijsenaars S N M 1997 First order analytic difference equations and inegrable quantum systems $J$. Math. Phys. 38 1069-146

[117] Ruijsenaars S N M 2000 On Barnes' multiple zeta and gamma functions Adv. Math. 156 107-32

[118] Sakai H 2001 Rational surfaces associated with affine root systems and geometry of the Painlevé equations Commun. Math. Phys. 220 165-229

[119] Satsuma J, Kajiwara K, Grammaticos B, Hietarinta J and Ramani A 1995 Bilinear discrete Painlevé-II and its particular solutions J. Phys. A: Math. Gen. 28 3541-8

[120] Schiff J L 1993 Normal Families (New York: Springer)

[121] Shimomura S 1981 Entire solutions of a polynomial difference equation J. Fac. Sci. Univ. Tokyo IA $28253-66$

[122] Shohat J A 1939 A differential equation for orthogonal polynomials Duke Math. J. 5 401-17

[123] Steinmetz N 1988 A uniqueness theorem for three meromorphic functions Ann. Acad. Sci. Fenn. AI 13 93-110

[124] Valiron G 1931 Sur la dérivée des fonctions algébroïdes Bull. Soc. Math. France 59 17-39

[125] Valiron G 1952 Fonctions Analytiques (Paris: Press. Univ. de France)

[126] Veselov A P 1992 Growth and integrability in the dynamics of mappings Commun. Math. Phys. 145 181-93

[127] Vojta P 1987 Diophantine Approximations and Value Distribution Theory (Lecture Notes in Math. vol 1239) (Berlin: Springer)

[128] Whittaker J M 1964 Interpolatory Function Theory (Cambridge Tracts in Mathematics and Mathematical Physics vol 33) (New York: Stechert-Hafner)

[129] Yanagihara N 1980 Meromorphic solutions of some difference equations Funkcialaj Ekvacioj 23 309-26

[130] Yanagihara N 1982 Meromorphic solutions of some difference equations of higher order Proc. Japan Acad. A 58 21-4

[131] Yanagihara N 1985 Meromorphic solutions of some difference equations of the $n$th order Arch. Ration. Mech. Anal. 91 169-92

[132] Yosida K 1933 A generalization of Malmquist's theorem J. Math. 9 253-6 